\title{
Sensory guided associative learning in paediatric migraine without aura
}

\section{$\mathrm{PhD}$ Thesis}

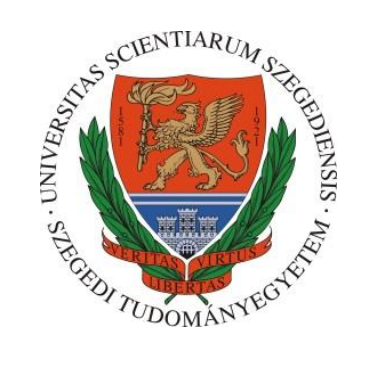

Giricz Zsófia, MSc

Supervisor: Dr. habil. Nagy Attila

\section{DEPARTMENT OF PHYSIOLOGY, FACULTY OF MEDICINE, UNIVERSITY OF SZEGED, 2020}




\section{Contents}

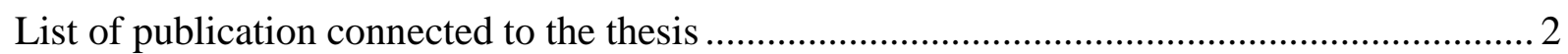

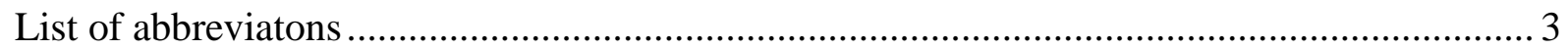

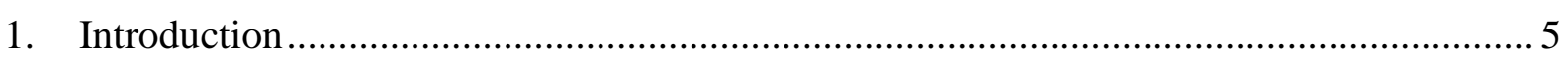

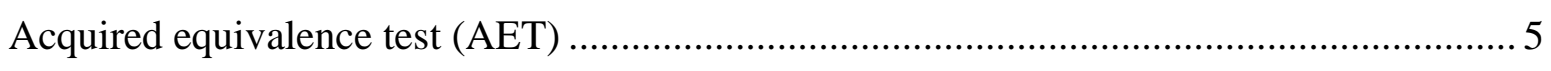

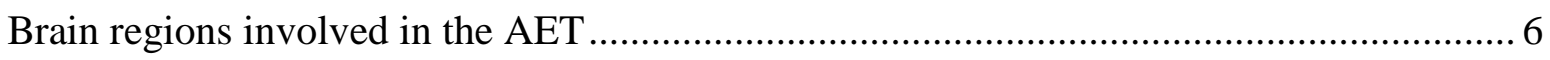

Migraine: epidemiology and clinical background ................................................... 10

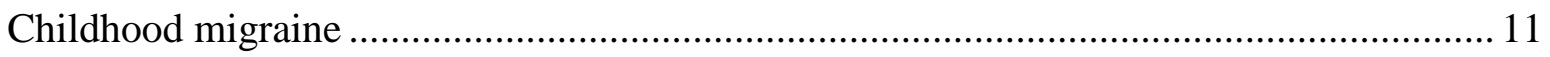

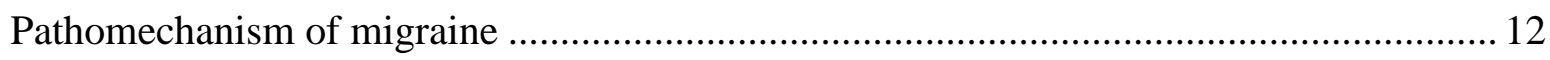

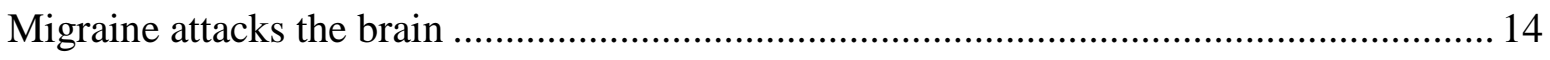

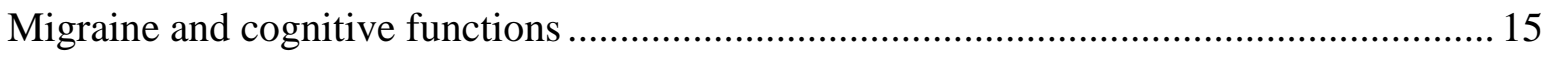

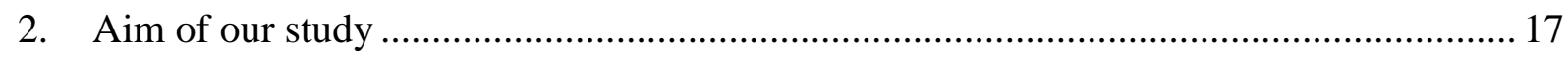

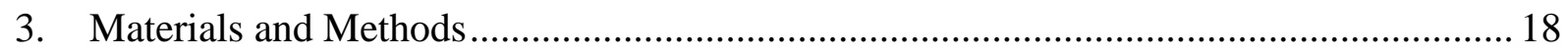

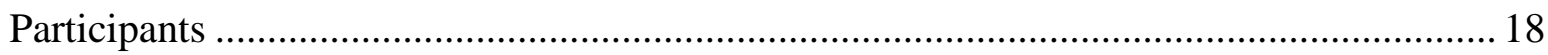

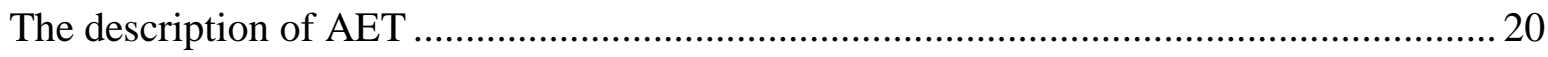

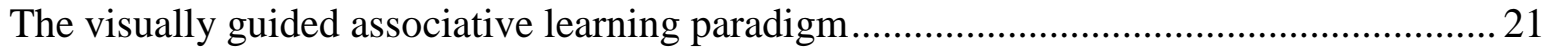

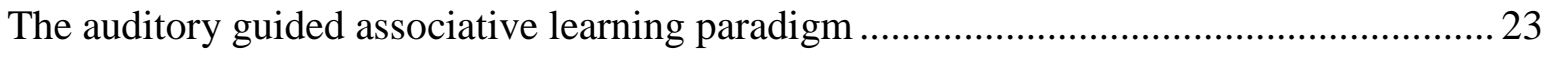

The multisensory guided associative learning paradigm.............................................. 24

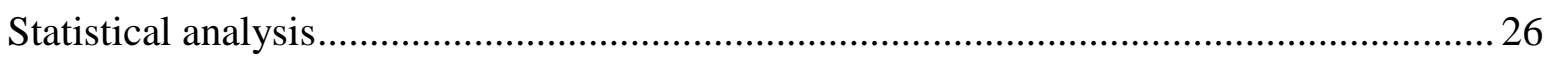

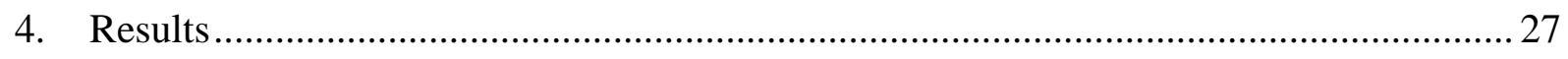

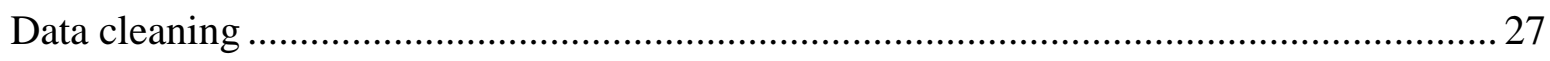

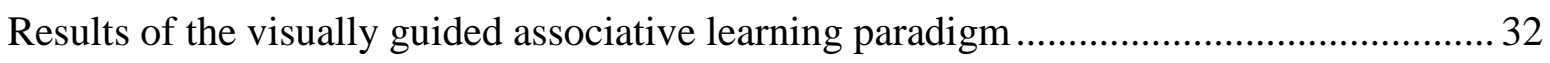

Results of auditory equivalence learning paradigm .................................................... 36

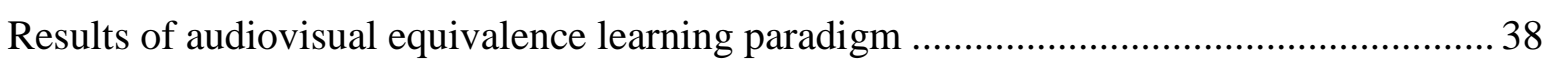

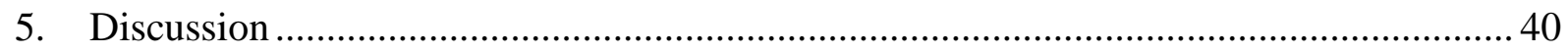

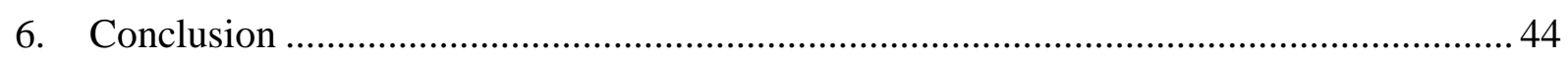

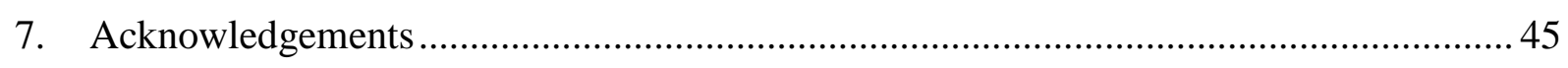

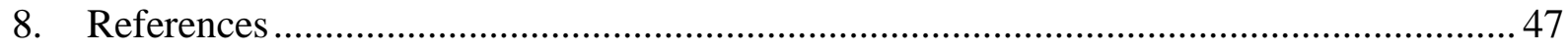




\section{List of publication connected to the thesis}

1. Zsófia Giricz, Ákos Pertich, Attila Öze, András Puszta, Ágnes Fehér, Gabriella Eördegh, Jenő Kóbor, Katalin Bihari, Éva Pálinkás, Gábor Braunitzer, Attila Nagy (2020) Visually guided associative learning in pediatric and adult migraine without aura. DOI: 10.1177/0333102420958388

Other publications:

1. Ágnes Fehér, Zsófia Giricz, Anna Juhász, Magdolna Pákáski, Zoltán Janka, János Kálmán (2018) ABCA1 rs2230805 and rs2230806 common gene variants are associated with Alzheimer's disease. Neurosci Lett. 2018 Jan 18;664:79-83.

2. András Puszta, Ákos Pertich, Xénia Katona, Balázs Bodosi, Diána Nyujtó, Zsófia Giricz, Gabriella Eördegh \& Attila Nagy (2019) Power-spectra and cross-frequency coupling changes in visual and Audio-visual acquired equivalence learning. Scientific Reports volume 9, Article number: 9444 (2019).

3. Gabriella Eördegh, Ákos Pertich, Zsanett Tárnok, Péter Nagy, Balázs Bodosi, Zsófia Giricz, Orsolya Hegedűs, Dóra Merkl, Diána Nyujtó, Szabina Oláh, Attila Öze, Réka Vidomusz, Attila Nagy (2020) Impairment of Visually Guided Associative Learning in Children With Tourette Syndrome. PLoS One 2020 Jun 16;15(6):e0234724.

4. András Puszta, Ákos Pertich, Zsófia Giricz, Diána Nyujtó, Balázs Bodosi, Gabriella Eördegh, Attila Nagy (2020) Predicting stimulus modality and working memory load during visual- and audiovisual-acquired equivalence learning. Frontiers in Human Neuroscience, DOI: 10.3389/fnhum.2020.569142 


\section{List of abbreviatons}

\begin{tabular}{|c|c|}
\hline AER & association error ratio \\
\hline AET & acquired equivalence test \\
\hline BG & basal ganglia \\
\hline CGRP & calcitonin gene-related peptide \\
\hline $\mathrm{CN}$ & caudate nucleus \\
\hline CSD & cortical spreading depression \\
\hline EEG & electroencephalogram \\
\hline fMRI & functional magnetic resonance imaging \\
\hline GER & generalization error ratio \\
\hline Gpe & globus pallidus the external segments \\
\hline Gpi & globus pallidus the internal segments \\
\hline GM & grey matter \\
\hline ICHD-II & International Classification of Headache Disorders, 2nd edition \\
\hline ICHD-3beta & International Classification of Headache Disorders, 3rd edition \\
\hline $\mathrm{Mg}^{2+}$ & magnesium \\
\hline MTL & medial temporal lobe \\
\hline NAT & number of acquisition trials \\
\hline PAG & periaqueductal grey \\
\hline PD & Parkinson's disease \\
\hline RAET & Rutgers Acquired Equivalence Test \\
\hline RER & retrieval error ratio \\
\hline SD & standard deviation \\
\hline $\mathrm{SN}$ & substantia nigra \\
\hline $\mathrm{SNr}$ & substantia nigra pars reticulate \\
\hline $\mathrm{SNc}$ & substantia nigra pars compacta \\
\hline
\end{tabular}


SP

substance $\mathrm{P}$

STN subthalamic nucleus

TGVS trigeminovascular system

WM white matter 


\section{Introduction}

\section{Acquired equivalence test (AET)}

Associative learning, a form of learning based on the formation of relationships between unrelated items. This relationship can usually develop as a result of learning based on similar or opposite characteristics, which coincide in time and space $[1,2]$.

Myers et al. [3] have developed a paradigm to investigate a specific kind of associative learning, called visually guided equivalence learning or face-fish paradigm. In this paradigm associations need to be acquired between antecedent stimuli (cartoon characters) and consequent responses (drawings of fishes) through trial and error.

The test is structured into sections including the acquisition phase (a feedback-guided learning of face-fish associations), the retrieval part (recalling the already acquired associations without feedback), and the generalization part (application of associative knowledge in new situations). In the acquisition phase participants need to learn which fish belongs to which character through trial and error learning. In the test phase the subjects need to recall the associations without feedback and they also have to apply their knowledge in new situations. Furthermore, they also have to realize that some stimuli are equivalent in regard to the associated consequents, as the same fish belongs to different cartoon characters. This equivalence is a functional equivalence because the stimuli are grouped not according to their basic features, but on functional characteristics [4]. Feedback-guided associative learning of the stimulus-consequent pairs and learning of the stimulus categories based on the hidden acquired equivalence of stimuli are both involved in the acquisition phase.

Earlier functional imaging studies revealed that with this paradigm, we could examine separately the medial temporal lobe (MTL) along with the hippocampus and the basal ganglia (BG) related learning and memory. The MTL plays an important role in declarative memory (intentional recollection of previous information) in contrast to the BG which is responsible for the procedural and habitual learning (such as how to ride a bicycle or how to brush your teeth) in humans and in vertebrates $[3,5]$.

Clinical studies with this paradigm could also dissociate the hippocampal- and basalganglia connected learning deficits. In Parkinson's disease (PD) the loss of dopaminergic neurons causes (beyond the well-described motor symptoms) several impairment in the cognitive domain such as the stimulus-response based habit learning [6, 7]. Earlier studies 
denoted that in the acquired equivalence test the PD patients performed significantly worst in the acquisition phase, but transferred the previously learned information as well as the control group [3]. In contrast, the first part of the paradigm did not cause any difficulty for the patients with mild to moderate hippocampal atrophy, but during the generalization part the pairing was not successful $[3,8,9]$.

This means that the BG is primarily involved in the initial phase of the task, while the hippocampus is responsible for generalizing the previously learned information (Figure 1. [3, 10]).

\section{Acquired Equivalence Test}

Aqcuisition Part

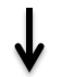

Frontostriatal loops
Retrieval and Generalization Part

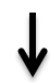

Medial tempral lobe (Hippocampus)

Figure 1. Schematic representation of the Acquired Equivalence Test (AET). The AET contains two phases: the acquisition and the test (retrieval and generalization) Part. The frontostriatal system is responsible for the acquisition and the medial temporal lobe including the hippocampus has an important role in the test phase.

\section{Brain regions involved in the AET}

The role of $\mathrm{BG}$ in the motor control (such as regulating muscle tone and voluntary movements) is intensively studied. It is also known (but for a less time) that BG is also crucial in several emotionally motivated movements, cognitive and effective functions. The system of BG consists of five functionally and anatomically linked subcortical nuclei. The medially located caudate nucleus $(\mathrm{CN})$ and the lateral putamen together form the neostriatrum. The other members that make up the system of BG are the globus pallidus (the external (Gpe) and internal (Gpi) segments), the substantia nigra (SN; pars reticulata ( $\mathrm{SNr})$ and compacta $(\mathrm{SNc})$ ), and the subthalamic nucleus (STN) [11]. 
The BG plays an important role in the integration of information between the thalamic and cortical areas. The pathways of the BG start from the cortex and return there, so they can also be called 'cortico-basal ganglia-cortical' loops. The main input structures of the BG are the neostriatrum and the STN, the information comes from the whole cerebral cortex. The processed information enters the motor thalamus through the $\mathrm{SNr}$ and GPi (the output structures) and then to the frontal cortex or limbic system. Furthermore, $\mathrm{SNr}$ projects into the superior colliculus and the pendunculopontine tegmental nucleus also. Cortico-BG-thalamocortical loops can be distinguished as functional units operating parallel to each other but not independently of each other. They all carry different information and move segregated side by side throughout. Based on the functional relationships formed by the input and output nuclei, we differentiate between direct, indirect, and hyperdirect loops (Figure 2. [12-15].

The parallel loops are well separable functionally and anatomically: motor loop (controlling of the skeletal muscle function, which is an unconscious regulation), oculomotor loop (responsible for the eye movements and the saccadic eye movements), dorsolateralprefrontal, lateral-orbitofrontal, and anterior cingular loops. The latter three are proven to be involved in the habit and the associative learning processes [16].

The appropriate operation of parallel pathways of the BG is a critical point, in the case of deviations from the normal case, the balance is upset, and motor changes can be observed. Degenerations of the neurons of the BG lead to different motor system diseases. Huntington's disease and the connected hyperkinetic disorders develop when the neurons in the striatum are damaged. While hypokinetic disorders, such as Parkinson's disease, are due to altered activity of dopamine-synthesizing neurons in the SNc $[11,17]$. Furthermore, the dysfunction of BG and frontal cortex circuits can be observed in patients with Tourette's syndrome or tics [18]. The dysfunction of neuronal circuits interconnecting the orbitofrontal cortex, $\mathrm{CN}$ and thalamus plays an important role in the pathogenesis of the obsessive-compulsive symptoms [19]. 


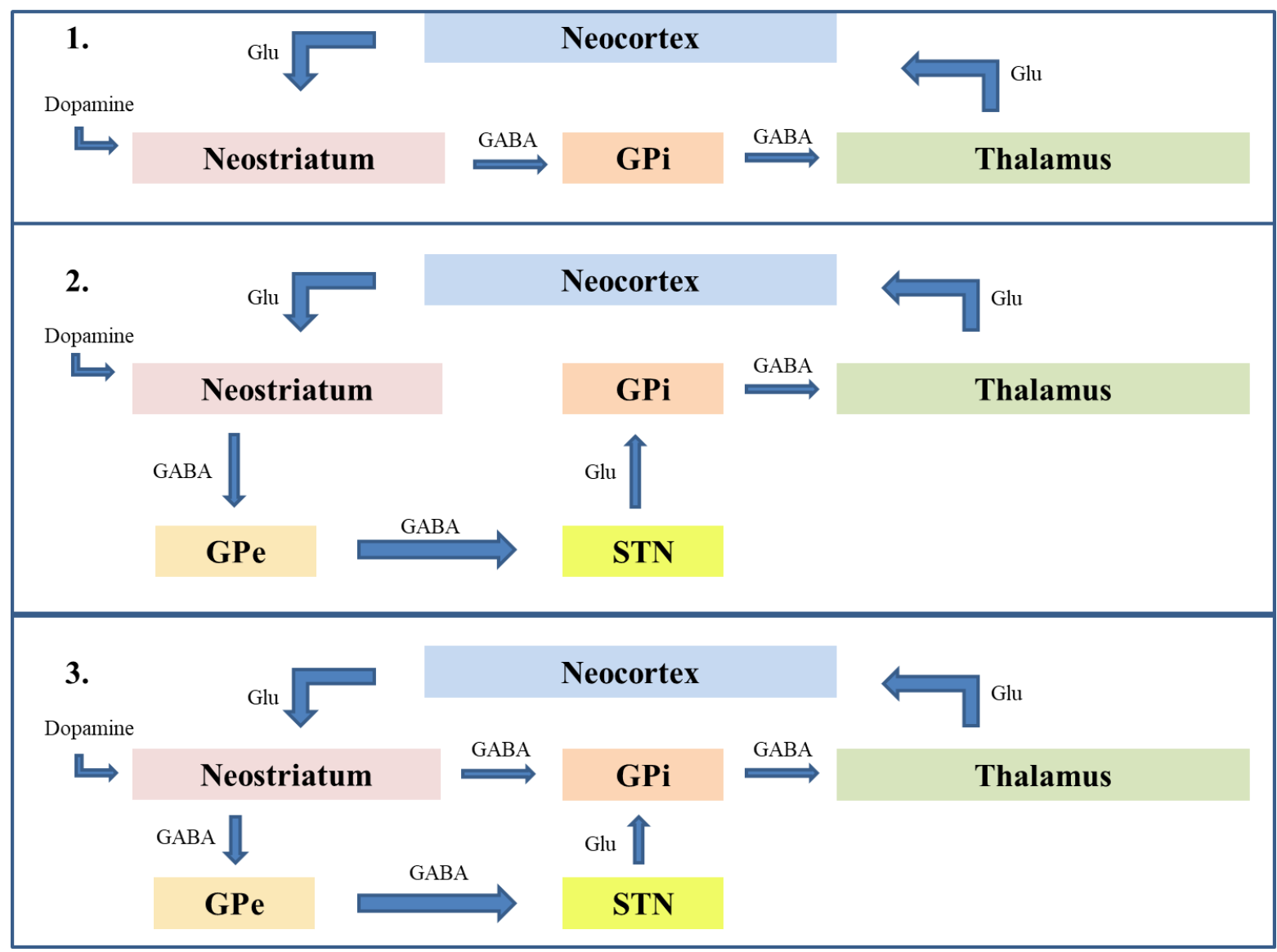

Figure 2. The basal ganglia pathways [11] 1. The direct pathway: increased activity of the cortex through inhibition releases the thalamus from inhibition, resulting in the activity of neurons from the thalamus to the motor cortex (release the movement). 2. The indirect pathway: The result is inhibition of the thalamus, which directly results in the ending of the flow of information between the thalamus and the cortex (inhibition of movement). 3. Balance between the two systems. Dopamine can modulate the neurons in the striatum. (The hyperdirect pathway is not shown in this figure). Glu: glutamate (excitatory neurotransmitter); GABA: Gamma-aminobutyric acid (inhibitory neurotransmitter); GPe: external globus pallidus; GPi: internal globus pallidus; STN: subthalamic nucleus

The MTL is an important component of the long-term memory. The central part of MTL memory system is the hippocampal region (Cornu Ammonis /CA/ fields 1-4, dentate gyrus and subiculum) and the anatomically related cortices: the perirhinal, entorhinal and parahippocampal cortex $[20,21]$ (Figure 3). The main function of MTL is to establish declarative memory by establishing a reciprocal relationship with the neocortex, but this feature is only temporary. Patients with histological evidence of hippocampal damage have 
moderate memory impairment $[22,23]$. It was observed in nonhuman primates that memory functions with limited hippocampal lesions were less impaired than when the lesions affected both the hippocampal region and the adjacent cortex [24].

The hippocampus has a prominent role in the process of explicit memory. It is responsible not only for explicit encoding, but also for recall, spatial orientation, and some implicit processes. The hippocampus also has a role in procedural learning as well as in recognizing and recalling complex correlations [25]. It processes information from all sensory areas, so it also plays an essential role in visual learning and the behaviour [5, 26].

MTL damage can be observed in many neuropsychiatric diseases. Hippocampal atrophy in Alzheimer's disease is associated with memory impairment [27]. Impairment of the MTL causes deficiency in episodic memory, intact working memory and remote autobiographical memory [28-30].

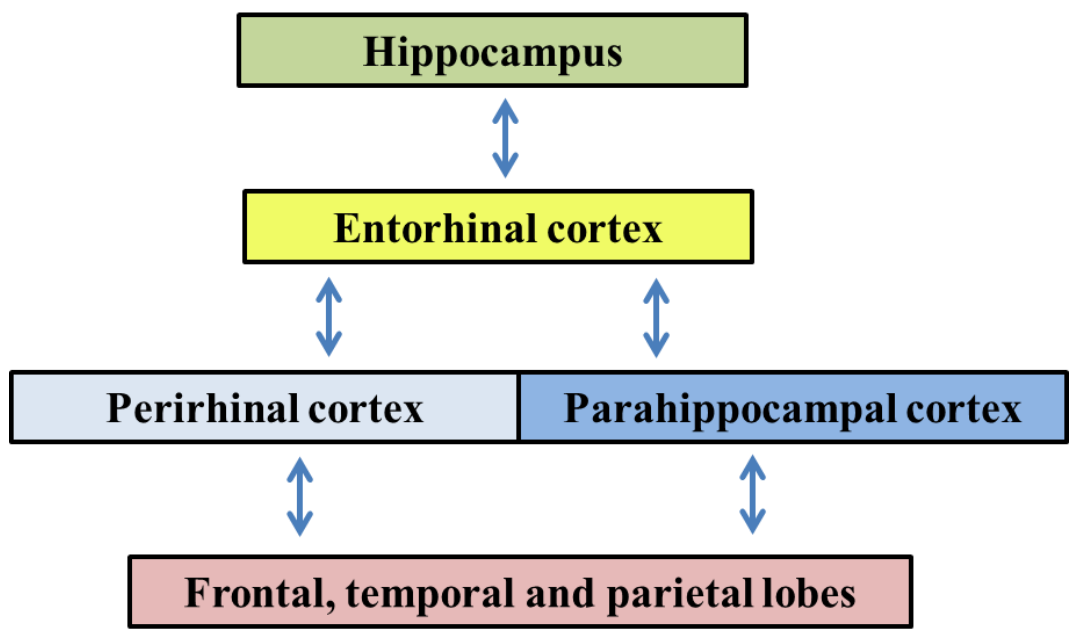

Figure 3. The medial temporal lobe structures in declarative memory [20]. The most influential input of entorhinal cortex comes from the adjacent perirhinal and parahippocampal cortex, which information comes from the unimodal and polymodal association areas (frontal, temporal and parietal lobes). In addition, the entorhinal cortex receives also other direct inputs from different cortices (orbital frontal cortex, cingulate cortex, insular cortex, and upper temporal gyrus). The main source of information in the hippocampus is the entorhinal cortex. All projections are reciprocal. 


\section{Migraine: epidemiology and clinical background}

Based on the $3^{\text {rd }}$ beta edition of the International Classification of Headache Disorders (ICHD-3beta) by the International Headache Society [31], we could classify headaches into two broad categories: the primary and secondary headaches. Primary headaches are determined by the headache and the characteristic clinical symptoms. The migraine, the tension-type headache and the trigeminal autonomic cephalalgias (including cluster headaches) are the most well-known primary headaches. Migraine can be divided into subtypes, from which the migraine without aura and the migraine with aura or the episodic and chronic migraine are the most common ones. The chronic form is characterized by 15 or more headaches per month for 3 consecutive months, of which at least 8 are migraine headaches. The appearance of migraine has a major impact not only on the lives of patients but also on their families as a whole [32].

Globally, $11 \%$ of the adult population suffer from migraine [33, 34], which by 2010 already became the 3rd most prevalent disease [31]. In childhood, the male:female ratio is 1:1, and after puberty, the sex ratio is gradually shifted and the male to female ratio changes to 1:3 in the early 40s $[33,35]$. It is more common among women aged 20-50, with a prevalence of 20-25\%. In Hungary, the number of migraineurs is estimated at 1.2-1.6 million [36, 37].

Migraine and the headache attacks can be divided into four main stages: prodroma, aura, headache and recovery (postdroma) [31]. Attacks are started with specific premonitory symptoms, that reflect mental (mood changes, difficulty concentrating), neurogenic (photophobia, phonophobia), and general conditions (tiredness, yawning, food cravings) develop [38, 39]. Aura is a sign of migraine that can represent approx. $30 \%$ of the cases and develops within 5 to 20 minutes and generally lasts for 60 minutes. Migraine aura symptoms consist of visual (scotoma, scintillating scotoma, tunnel vision or visual hallucinations), sensory (limb paraesthesia), motor abnormalities (coordination disorder), speech disorder, brainstem dysfunction (vertigo, nausea), or a combination of them. These signs are followed by a headache either immediately or in less than an hour [40-42].

According to the ICHD-3 beta migraine is characterized by the attacks lasting 4 to 72 hours, which may appear several times per month, and commonly present of at least two of the following: unilateral location, pulsating quality, and aggravation by or causing avoidance of routine physical activity. Nausea, vomiting, photophobia, and phonophobia are common accompanying symptoms [31]. It is best for a patient to lie down in a silent, dark room. 
Physical activity worsens the condition. After migraine attack which usually resolves gradually, patients are tired, irritable, inattentive, unable to concentrate. Some patients become euphoric when pain is gone, while others become depressed. Patients usually return to their usual daily activity 24 hours after the pain [43, 44].

\section{Childhood migraine}

Migraine is most common between the ages of 20 and 45, but affects children as well. The majority of paediatric patients with recurrent headaches suffer from primary headaches being migraine the most common one $[45,46]$. The migraine without aura is the most frequent form of migraine in children and adolescents (60-85\%) [47]. Diagnosis of childhood migraine is challenging because of its varying symptoms, triggers and aetiology. Trigger factors are not the cause, they only provoke the attacks. The childhood migraine is often misdiagnosed, because the pain is not limited to the head, but many times occur as abdominal symptoms or vertigo [48, 49]. The manifestation of migraine may also be connected to children's lifestyle, the psychological stress, the excessive physical fatigue and the exposure to strong light effects $[50,51]$. The occurrence of migraine is profoundly influenced by female sex hormones in puberty. In a previous study demonstrated that female sex steroids play a permissive role in the vascular and neural changes that characterize migraine [52].

Paediatric migraine without aura is characterized by moderate or severe intensity attacks within the range of 1-72 hours in duration. Furthermore, all of the attacks share at least two of the following characteristics: they can be unilateral or bilateral (children younger than 15 years of age), pulsating or throbbing in quality, aggravated by routine physical activity. During the migraine attacks at least two of the following symptoms can occur: nausea and/or vomiting, photophobia or phonophobia [31, 47]. Of particular note is that the migraine attacks stop, when the children fall asleep. Pediatric migraine headache is shorter in duration, more often bilateral compared to symptoms of adult patients, and it is usually frontotemporal but occipital headache in children is rare [31].

The prevalence of childhood migraine varies with gender and age: under the age of 7 it is $2.5 \%$ and both genders are affected equally, from age of 7 to puberty it is $5 \%$ with a $3: 2$ female-male ratio, while it is $5 \%$ in post-pubertal boys and $10 \%$ in post-pubertal girls. Migraine headaches occur more frequently earlier in boys, but during the puberty, the 
characteristic 2-3:1 female to male ratio is reached. The incidence of migraine decreases after puberty, but the gender ratio remains unchanged $[53,54]$.

Several conditions have a comorbid relationship with migraine, such as psychological or emotional disorders (depression, anexiety), sleep disorders and obesity [55-57]. These additional disorders could make the diagnosis and treatment of migraine hard.

\section{Pathomechanism of migraine}

The pathomechanism of migraine is not well-known, although the results of the past two decades have enriched our knowledge. The migraine attacks are probably caused by a combination of several changes (Figure 4.). The results of modern molecular and functional techniques have broken down the rigid boundaries between the vascular (focus on the role of vessel tone) and neurogenic (concentrate on peripheral nerve sensitization) theories [58] and have led to the development of a new common neurovascular approach [59-61]. According to this, headache is associated with activation of primary afferents, which release inflammatory mediators, causing plasma protein extravasation and then neurogenic inflammation. However, the mechanism, how primary afferents are activated, is unclear.

It has been observed that during spontaneous migraine attacks the dorsolateral pons [62] and the dorsal part of the midbrain $[63,64]$ are activated. The changes in the activation of the raphe magnus, the nucleus raphe dorsalis, the locus coeruleus and the periaqueductal grey (PAG) leads to migraine attacks, which is the basis of the migraine generator theory [62]. All migraine generator nuclei are members of the endogenous pain control pathways, which also show a subnormal activity during the migraine headache [65].

Previous studies have reported that the trigeminovascular system (TGVS) plays a key role in the process of migraine [59, 65-68]. Pain-sensitive structures, such as the meningeal arteries and the dura mater, receive nociceptive sensory innervation from the ophthalmic branch of the trigeminal nerve. The majority of the trigeminal neurons release vasoactive mediators, such as calcitonin gene-related peptide (CGRP), neurokinin A and substance P (SP), resulting in vasodilation of the blood vessels and plasma protein leakage from the dural vessels. The vasoactive mediators are also responsible for the neurogenic inflammation, which is thought to play an important role in the pain during the migraine attacks [69-71]. 
Furthermore, the effect of CGRP and SP may result in the peripheral sensitization of the primary afferent neurons [65].

The release of the CGRP from the meningeal nociceptors, the activated mast cells and the excited TGVS can trigger the cortical spreading depression (CSD) [72]. It is a unique, migraine-specific wave of excitatory neural activity in the cortex, the cerebellum and the hippocampus. During the CSD increased nitric oxide and glutamate release can be observed [73]. The excitatory activity and the hyperaemia in the cortex is followed by a depressed state and decreased cortical blood flow $[74,75]$. The CSD starts from the occipital cortex and spreads in the rostral direction at a speed of 2-6 mm/min. Previous studies $[76,77]$ have accepted the CSD only as the cause of migraine aura symptoms. Furthermore, the experimental studies have demonstrated the process, which possibly connect the CSD and the activated TGVS during the development of lateralized head pain [76, 77].

Several migraine researches have shown disturbances in ion homeostasis. Magnesium $\left(\mathrm{Mg}^{2+}\right)$, which is one of the most important intracellular cations, plays a key role in the organic cellular processes. The earlier findings are lowered ionized $\mathrm{Mg}^{2+}$ levels in the serum and the brain during the migraine. The low level of $\mathrm{Mg}^{2+}$ links to the hyperexcitability of the cortex, cerebrovascular constriction and may also promote cortical spreading depression $[78,79]$.

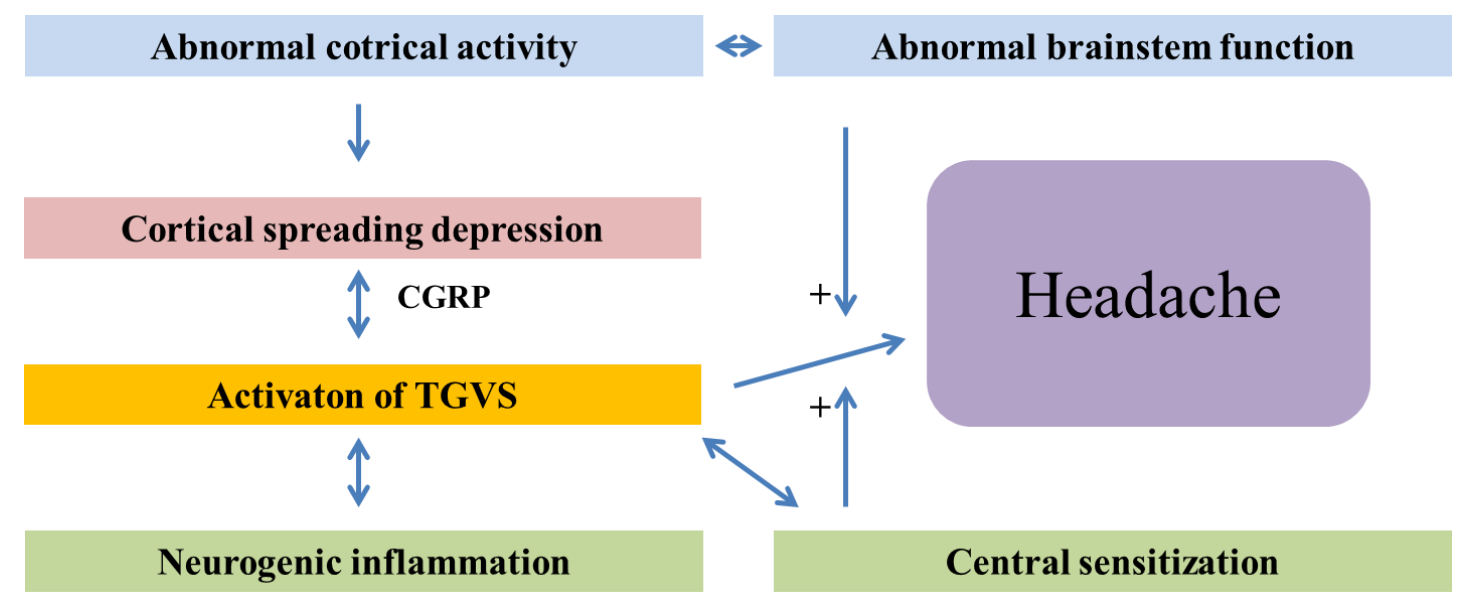

Figure 4. Pathophysiology of migraine headache. Migraine headaches are caused by altered function of cortex and brainstem. The first two steps in developing migraine with aura and perhaps also migraine without aura are the appearance of cortical spreading depression and the activation of the trigeminovascular system (TGVS). The calcitonin gene-related peptide (CGRP) is the key molecule in the development of the migraine pain [80]. 


\section{Migraine attacks the brain}

Recently, several imaging studies have addressed the effects of migraine on the brain. Increased cortical excitability [81-83], altered brain blood flow [84, 85], and changes in the pain modulatory system were observed $[86,87]$. Numerous brain imaging studies of migraine patients were found increased grey matter (GM) density in the PAG and the dorsolateral pons $[88,89]$ and reduced GM in the frontal, temporal and occipital lobe, as well as in the anterior cingulate cortex, the superior frontal gyrus and limbic lobe, the basal ganglia and the primary and secondary somatosensory cortices [88-92]. Furthermore, the decreased GM was described in the pain-related brain regions, but these lesions proved to be reversible as the number of migraine attacks decreased [93]. DaSilva et al. [94] described increased GM thickness in the somatosensory cortex, and another study reported an increase in GM thickness in visual motion processing areas in the migraineurs using surface-based morphometry [95]. In contrast, Maleki and co-workes [96] found significantly thicker cortex in the precuneus and posterior insula in patients suffering from migraine.

Several studies reported the involvement of BG in the migraine. One study reported abnormal volume and resting-state networks of the left caudate and right nucleus accumbens in the migraineurs [97]. Furthermore, other imaging studies have found decreased activation in the BG due to migraine [98] and increased blood flow during the episodes in the migrainers' BG [99]. In addition, significant changes in the structure and function of the BG are observed as a result of recurrent migraine pain [100].

In addition to changes in $\mathrm{BG}$, several alterations in the hippocampus as a result of migraine have also been described. Decreased volume was observed in the hippocampus in newly diagnosed migraineurs [92]. In contrast, Maleki et al [101] have found differences in the volume between the low and high headache frequency: significant greater bilateral volume was observed in the patients with low headache frequency compared to the migrianeurs with high headache frequency. Moreover, one study reported larger hippocampal (and also other pain-processing regions) nociceptive activation during thermal pain stimulation in the migraine using functional magnetic resonance imaging (fMRI) [102], which seems to be correlated with headache frequency. 
The changes in white matter (WM) are not so clear: some studies have found deep white matter lesions [103], frontal cotrical atrophy and axonal loss [104-106], while others have reported no significant differences in the WM in migraine brains by MRI research [92]. Negm and co-workers [107] have found WM hyperintensities in $43.1 \%$ of migraine patients, and this phenomenon is more profound in patients suffering from migraine with aura.

\section{Migraine and cognitive functions}

There are very few studies available that examine the cognitive functions of migraineur patients and not all studies have described the cortical deficits or deterioration in cognitive performances. Leijdekkers et al. [108] and Mulder and co-workers [109] found no significant differences between the adult controls and migraineurs testing the learning and memory functions. In contrast, several studies described impaired attention, cognitive and memory dysfunctions in adult migraineurs [110-112]. According to some studies, there is no difference in intelligence and processes requiring explicit memory between migraineurs and healthy subjects [113]. In adults, the visuo-cognitive processing has been shown not to be working properly in patients suffering from migraine, both with and without aura [114-116].

Braunitzer et al. [117] have found that migraine-suffering children exhibited a slower development of visual contour integration. Moreover, in paediatric migraine the contrast sensitivity is mostly affected at low spatial frequencies [118]. It has been also observed that motion coherence processing capacity was reduced [119]. The impairment of short-term and long-term memory in children suffering from migraine [120] and the significant differences in the mean total and verbal intelligence quotient scores between the child migraineurs and the control children were previously discovered [121]. Furthermore, in the previous studies the impairment of psychomotor abilities, attention and verbal memory, have been also found [122], which may be due to the damage caused by migraine. Comparing adolescent migraineurs and healthy groups using the Addenbrooke's Cognitive Test, the overall performance in the migraine group was significantly lower, resulting from significantly worse performance in the verbal fluency and memory tasks [123]. In our previous study, a poorer performance in associative learning and an extensive impairment in generalization was observed in adult migraineurs without aura. These findings seem to support beside the hippocampi the involvement of the basal ganglia in the pathogenesis of migraine [124]. 
According to Vuralli's findings, other neuropsychiatric comorbidities, like chronic pain and depression, arise in the background of cognitive dysfunction in migraine [125]. However, most of the cognitive differences in migraine cannot be obviously explained by the above. Also, this is supported by the fact that tension and cluster headaches are not associated with interictal cognitive abnormalities similar to migraine. Furthermore, migraine has been identified as a risk factor for late-onset dementia, within which it has been more closely associated with Alzheimer's disease. This finding highlights the chances of progressive cognitive decline and raises the need for early monitoring of migraineur patients for dementia [126]. 


\section{Aim of our study}

The aim of our study was to investigate whether the differences found in the AET performance among adults suffering from migraine compared to healthy age-matched controls can be observed in children migraineurs as well. We also intended to investigate the pediatric patients' performance compared to the control children groups in the auditory and multisensory guided equivalence learning developed and validated by our lab [127], based on AET.

The objectives of our study were:

- to examine the performance of the children migraineurs in the visually, in the auditory and in the multisensory guided equivalence learning and compared to the age-matched children controls

- to compare our previous results from adult migrianeurs and adult controls with results from pediatric migrianeurs and control children in the visually guided AET 


\section{Materials and Methods}

\section{Participants}

29 children diagnosed with migraine without aura between ages of 8 and 17.5 years and 22 adult migraine patients between ages of 20 and 52 years (published earlier in [124]) were enrolled in our study. One of them was excluded for signs of excessive anxiety during the procedure, another one failed to cooperate. This left us with the final sample of 27 patients. The study period spanned 18 months altogether, from January 2018 to November 2019 (the three months of the summer holiday season excluded in each year). The children patients were recruited from the Department of Pediatrics, University of Szeged, Hungary. The details of the recruitment of the adult patients and controls is described in Öze et al. [124], the adult migraineurs were patients of the Neurology and Stroke Department of the Hospital of Kecskemét, Hungary.

The diagnostic criteria of migraine without aura were established according to the International Classification of Headache Disorders, 2nd edition (ICHD-II). All children patients were diagnosed by the same pediatric neurologist, and also, all adult cases were diagnosed by the same neurologist. The inclusion criteria were the diagnosis of migraine without aura, while exclusion criteria were the presence of other neurological, psychiatric or ophthalmological disorders. At least five days had passed since the last attack at the time of testing for all patients and no attack occurred in the 24 hours following the testing.

The control group for children patients involved 27 healthy subjects matched on age, gender and level of intelligence tested by Raven's Progressive Matrices. In the migraineur and control child groups mean age ( \pm standard deviation (SD)) was $14.1 \pm 3$ years and $14.2 \pm 3$, and the female-male ratio was 15 girls and 12 boys in the visual paradigm (Table 1). In the auditory paradigm the female:male ratio was 13:11 and the two investigated groups mean age $( \pm \mathrm{SD})$ was $13.9 \pm 3$ years. In the audiovisual paradigm the female-male ratio was 10 boys and 12 girls and in the migraineur and control child groups mean age $( \pm \mathrm{SD})$ was $14.1 \pm 3$ years. The adult control group consisted of 22 healthy individuals matched on age, sex and level of education [124]. Adults participated only in the visual paradigm. In the adult migraineur group the mean age $( \pm \mathrm{SD})$ was $44 \pm 11.76$ years, in the adult control group it was also $40 \pm 11.76$ years and in both groups the female-male ratio was 20 women and 2 men (Table 1 ). 
The children controls were recruited from different primary schools and secondary schools in Szeged, Hungary. The participants in the child control group had no history of any kind of headache, and they were also free of any kind of neurological, psychiatric or ophthalmological disorder as well. Only participants without colour vision deficiency tested by Ishihara plates were eligible for this study in all investigated groups. The adult controls were recruited from the employees working at the Faculty of Medicine, University of Szeged, Hungary. The exclusion criteria were the same as in the child control group.

The study protocol conformed to the ethical principles of the Declaration of Helsinki in all aspects. Prior to testing, participants and their guardians were informed about the background, aims and procedures of the study both orally and in written form. None of the subjects received any compensation for their involvement, and they were informed that the study has merely scientific purposes without direct diagnostic or therapeutic use and they are free to leave at any time. All recruitment and protocols were conducted with written informed consent and with the approval of the Regional Research Ethics Committee for Medical Research at the University of Szeged, Hungary (52/2015).

Demographic and migraine-specific characteristics of the investigated groups are summarized in Table 1.

\begin{tabular}{|c|c|c|c|c|}
\hline & $\begin{array}{l}\text { Child } \\
\text { controls } \\
n=27\end{array}$ & $\begin{array}{l}\text { Child } \\
\text { migraineurs } \\
\mathrm{n}=27\end{array}$ & $\begin{array}{l}\text { Adult } \\
\text { controls } \\
n=22\end{array}$ & $\begin{array}{l}\text { Adult } \\
\text { migraineurs } \\
\mathrm{n}=22\end{array}$ \\
\hline Age (years, mean \pm SD) & $14.1 \pm 3.0$ & $14.2 \pm 3.0$ & $40 \pm 11.76$ & $40 \pm 11.76$ \\
\hline Female/male ratio & $15 / 12$ & $15 / 12$ & $20 / 2$ & $20 / 2$ \\
\hline $\begin{array}{l}\text { Migraine history (years, } \\
\text { mean } \pm \text { SD) }\end{array}$ & - & $\begin{array}{l}\text { freshly } \\
\text { diagnosed }\end{array}$ & - & $15.64 \pm 10.9$ \\
\hline $\begin{array}{ll}\text { Attack } & \text { frequency/month } \\
(\text { mean } \pm \text { SD }) & \end{array}$ & - & - & - & $5.0 \pm 4.8$ \\
\hline
\end{tabular}

Table 1. Demographic and migraine specific characteristics of the investigated groups in the visual paradigm. 


\section{The description of AET}

The tests were run on PC and with Sennheiser HD 439 closed, over-ear headphones for auditory and multisensory testing. Stimuli were presented and responses were collected using a personal computer with a 17-inch CRT screen. During testing, the subjects were sitting at a standard distance $(114 \mathrm{~cm})$ from the computer screen in a quiet room. The " $\mathrm{X}$ " and "M" keys of the keyboards were labelled "LEFT" and "RIGHT". One participant was tested at a time without forced quick responses or any time limit.

The testing of the visual associative learning paradigm (RAET) was carried out according to the method of Myers and co-workers [3]. The testing software originally written for iOS was slightly modified and translated to Hungarian, as well as reprogrammed in Assembly for Windows with the written permission of Catherine E. Myers (Rutgers University, NJ, USA). Beside the RAET, we also introduced an auditory and audiovisual guided learning test, implemented in Assembly for Windows [127, 128].

During the paradigms, the participants had to connect with the antecedents and consequent stimuli during in each trial of the tasks. The trials of the three paradigms were structured into two phases: 1 . the acquisition phase, 2. the test phase which can be divided in two parts, the retrieval and generalization parts. In the acquisition phase the participants had to learn the association of two sensory stimuli and indicate their choice in each trial by pressing the LEFT or RIGHT keys of the keyboard (Figure 5). The computer gave feedback, the green check appeared if the answer is correct, in contrast the incorrect answer was indicate red $\mathrm{X}$. New associations were introduced one by one during the association phase. In the test part of the paradigm, the participants had to recall the learned associations (retrieval part) and had to create new, hitherto not presented, but based on earlier learned pairs predictable, two pairs also appear (generalization part) without any feedback. The unknown new associations were shown randomly mixed among the previously learned pairs. The subjects had to achieve a certain number of consecutive correct answers after the presentation of each new association (4 after the presentation of the first association, and 4, 6, 8, 10, 12 with the introduction of each new association, respectively) to be allowed to proceed. This meat, that the participants could proceed to the next phase, only they learned the all associations in the first phase. From this also follows that the length of the association phases varied among the participants, depending upon how efficiently they learned. Thereafter, the test phase 
consistently contained 48 trials, 36 trials of previously learned associations (retrieval part) and 12 new, hitherto not shown, but predictable news (generalization part).
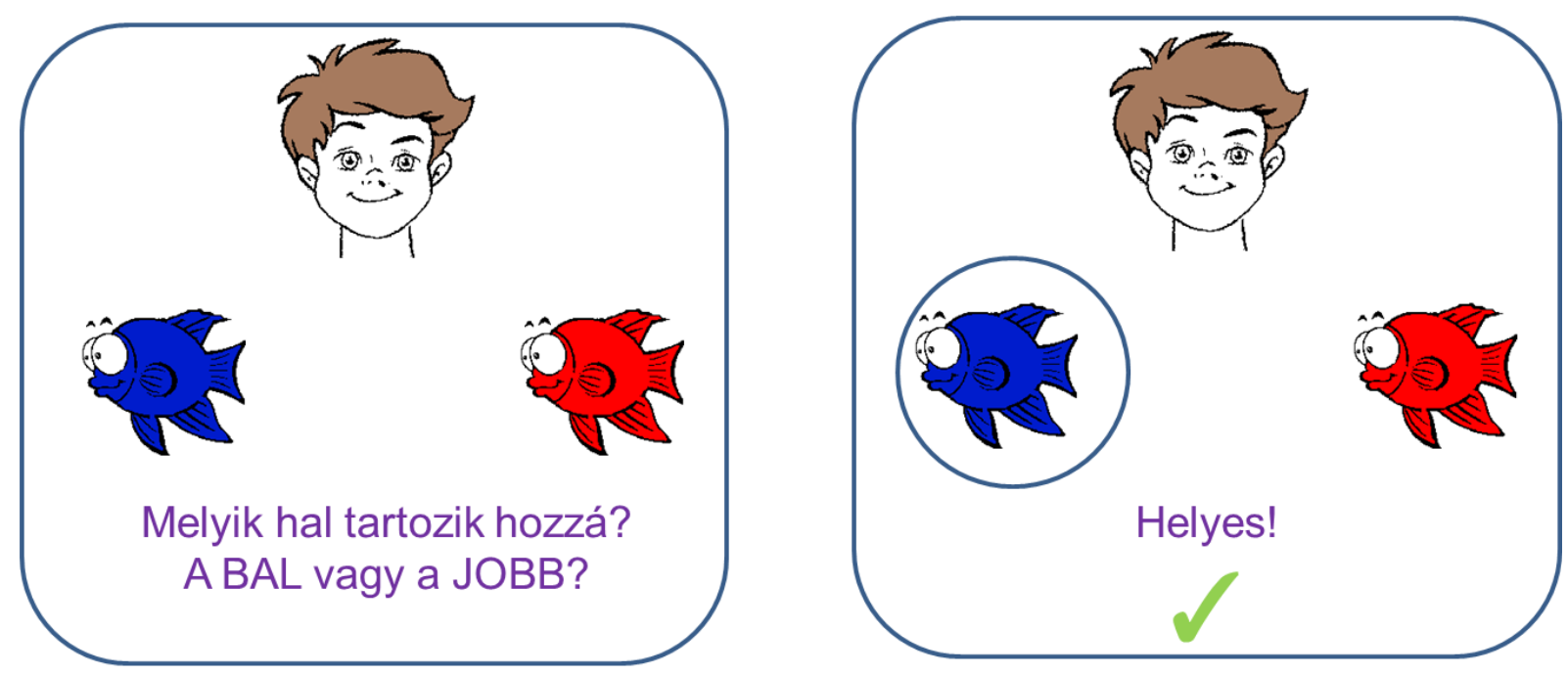

Figure 5. Two figures of the trials from the visual paradigm. Each trial included an antecedent stimuli (face) and two possible consequents (two different colour fishes). In the left picture text means: Which fish goes with this face? LEFT or RIGHT? In the right picture there is the correct answer with green check.

\section{The visually guided associative learning paradigm}

The visual stimuli referred to as antecedents were cartoon faces of a woman (A1), a girl (A2), a man (B1) and a boy (B2). The responses referred to as consequents were yellow (X1), red (X2), green (Y1) and blue (Y2) fish (Figure 6.). During a trial the participant saw a face (an antecedent stimulus) and a pair of fish of different colour (two possible consequents) and asked to give a choice by pressing on one of two buttons corresponding to the two fish of different colour.

During the acquisition phase participants learned a series of antecedent-consequent pairs via trial and error. When face A1 or face A2 were shown, the correct choice was X1 fish over Y1 fish; however, when B1 or B2 appeared on the screen, the correct answer was Y1 fish, instead of X1 fish. Thereby beside the face-fish associations, participants have also learned that the A1 face is equivalent with A2 face in relation to the associated fish. New associations were introduced gradually/incrementally (see in Figure 6), and they were 
presented mixed with trials of previously learned associations. In detail, 4 consecutive correct responses were required after the presentation of the first two associations (A1 and X1, B1 and $\mathrm{Y} 1)$, and an increasing number of consecutive correct choices $(6,8,10,12)$ were needed after the presentation of each new association in order to make sure that the participant successfully acquired each association before proceeding to the test phase.

In the test phase the task remained the same but feedback was no longer provided. The test phase consisted of 48 trials, as opposed to the 16 trials of the original paradigm. The participants got 48 tasks showing already known pairs (retrieval) mixed with tasks presenting new pairs (A2 and X2; B2 and Y2) testing the generalization of the learned equivalence, as opposed to the 16 trials of the original paradigm. Subjects were not informed about the appearance of new associations.

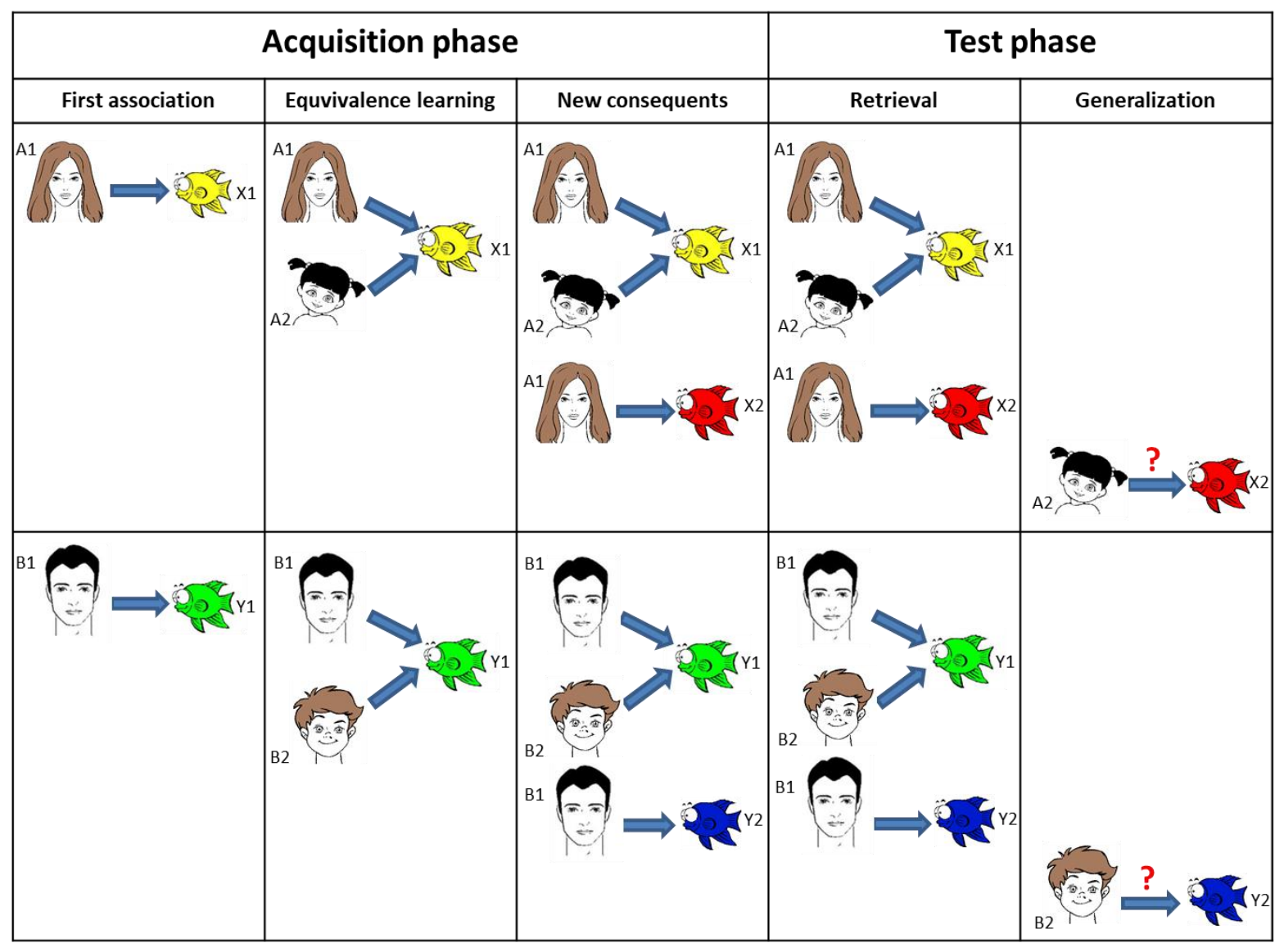

Figure 6. The antecedent-consequent pairs of the visually guided equivalence learning test (after Myers et al., 2003.). Antecedents (stimuli) were cartoon faces of a woman (A1), a girl (A2), a man (B1) and a boy (B2). Consequents (responses) were drawings of fish of yellow $(\mathrm{X} 1)$, red (X2), green (Y1) and blue (Y2) colour. 


\section{The auditory guided associative learning paradigm}

In the auditory paradigm, in contrast the visual and multisensory paradigm, every sound had to be associated with the corresponding button ( $\mathrm{X}$ as the LEFT, $\mathrm{Y}$ as the RIGHT button), not with the other sound. Four pairs of sounds (eight stimuli) were used: two animal sounds (a cat meowing and a dog barking, blue speaker), two different gender voices (a woman and a man said a Hungarian word, yellow speaker), two instrumental sounds (a note played by a guitar and a piano, green speaker), two sounds of vehicle (sounds of an ignition key and a motorcycle, pink speaker) (Figure 7). Each sound lasted $1.5 \mathrm{sec}$ long, and had same intensity $(60 \mathrm{~dB})$. The participants heard the pairs before the test began and we checked for proper functioning of the headphones. The different pairs of sounds were randomly presented.

During the acquisition part, the participants had to learn the distribution of sounds between the two buttons. The participants similarly to the visual paradigm received feedback in this phase. The first sound of the pair A1 (a cat meowing) could be associated to the LEFT button and the second sound of the same pairs B1 (a dog barking) to the RIGHT button. After that the participants have also learned that the A1 sound is equivalent with A2 sound (woman said a Hungarian word) in relation to the associated LEFT button and also the B1 sound is equivalent with B2 sound (a man said a Hungarian word) in relation to the connected RIGHT button. New associations were introduced incrementally (A3: sound of a guitar and B4: sound of a motorcycle), and they were presented mixed with trials of previously learned associations. In the test phase, without any feedback, the remaining two sounds (A4: sound of ignition key and B3: sound of a piano) were presented, and the participants had to generalize the new associations. 


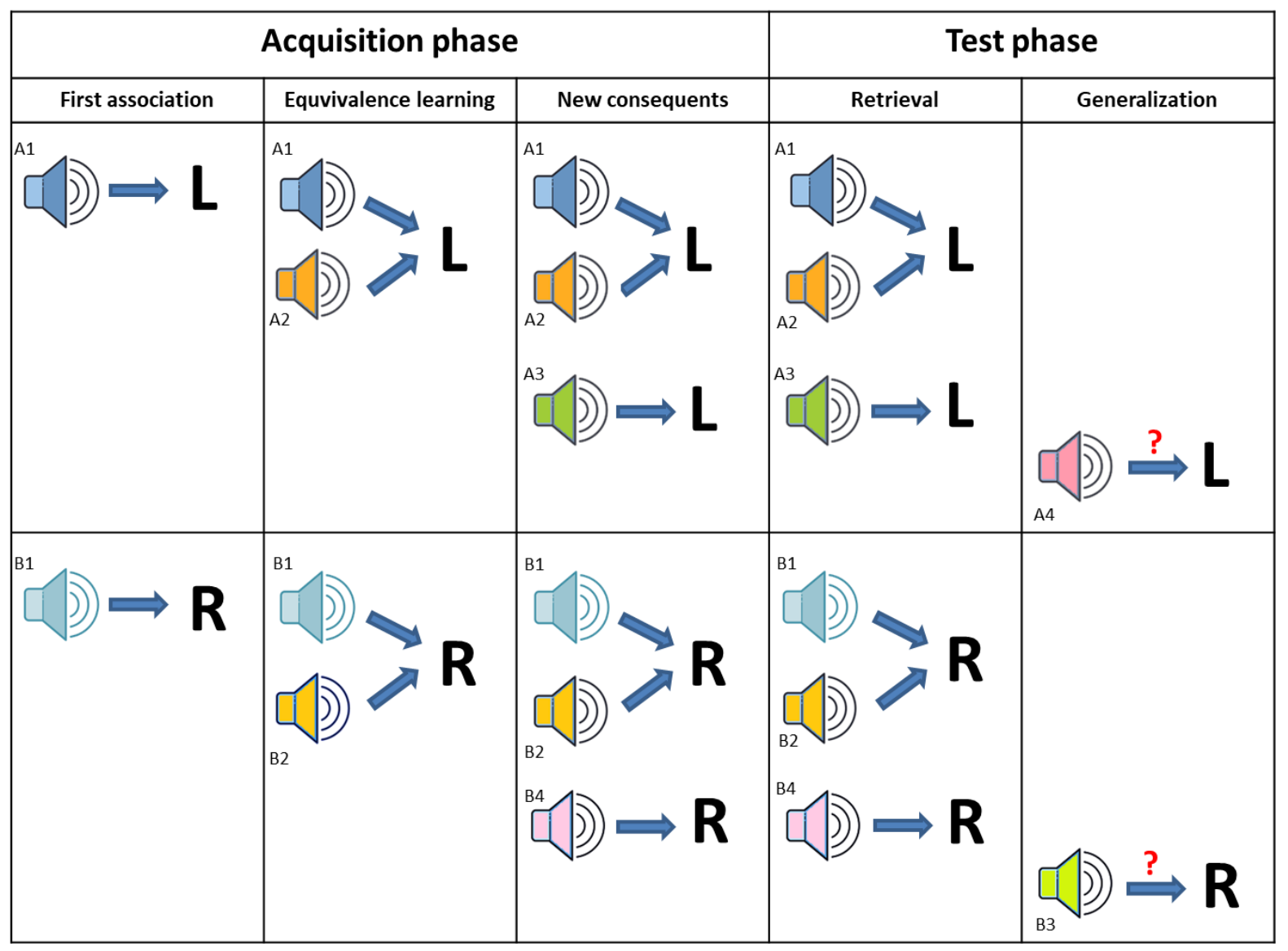

Figure 7. The auditory guided associative learning test. The colours of speakers symbolize the different pairs of sounds (blue: two animal sounds, yellow: two human voices of different gender, green: two sounds of musical instruments, and pink: two sounds of different vehicle).

\section{The multisensory guided associative learning paradigm}

The structure of the multisensory (audio-visual) paradigm was the same as the visual paradigm. The main task of the participants was to learn to associate four antecedent sounds (one of the pairs used in the auditory paradigm, e.g.: a cat meowing (A1), a man said a Hungarian word (A2), sound of a piano (B1) and a motorcycle sound (B2)) and the consequents four faces (as in the visual paradigm; a woman (X1), a girl (X2), a man (Y1) and a boy (Y2)) (Figure 8). In each trial a sound was played and two faces presented. During the acquisition phase, the participants had to learn which sound associate with which face. In this part six of the possible eight sound-face combinations were learned. Until this point, there was feedback about the correctness of decisions. After that in the test phase, without any 
feedback, beside the already acquired combinations (retrieval part), the test presented the new combinations also (generalization part). If the participants learned that the A1 and A2 sounds and B1 and B2 sounds are equivalent, they could generalize the rule and associated the A2 sound to the girl (X2), and B2 sound to the boy (Y2).

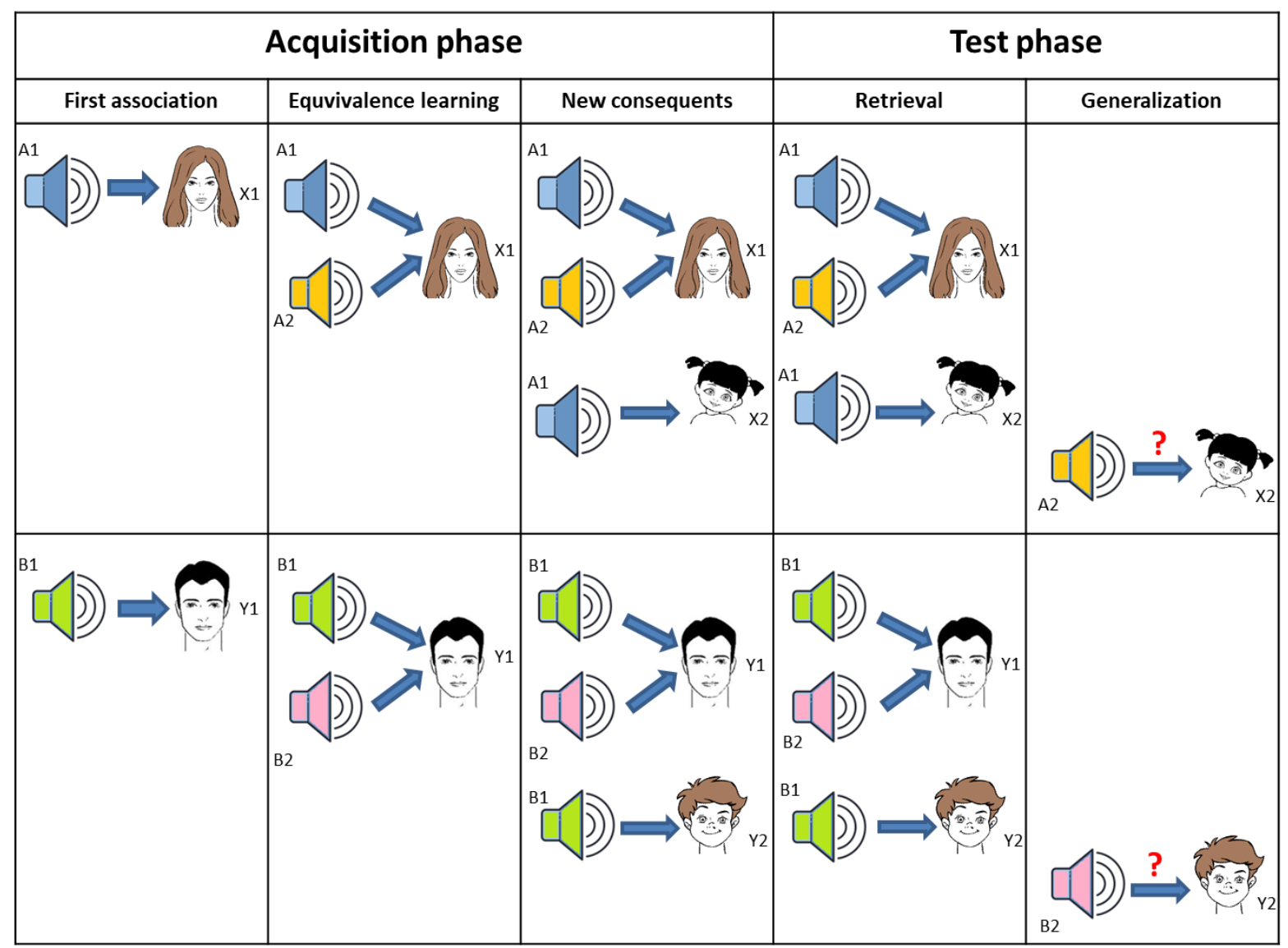

Figure 8. The antecedent-consequent pairs of the multisensory guided equivalence learning test. Antecedents (stimuli) were different sounds (A1, A2, B1, B2). Consequents (responses) were cartoon faces of a woman (X1), a girl (X2), a man (Y1) and a boy (Y2). 


\section{Statistical analysis}

Statistical analysis was performed in SPSS 21.0 (IBM, USA). The level of significance was set at $p=0.05$.

Participants were divided into four groups: child migraineurs, child controls, adult migraineurs and adult controls. The performance of the participants was characterized with four parameters: the number of trials necessary for the completion of the acquisition phase (NAT), association error ratio (the ratio of incorrect choices during the acquisition trials, AER), retrieval error ratio (RER), and generalization error ratio (GER).

Before the hypothesis tests, extreme outliers were removed from the dataset as suggested by Tukey [142]: for each variable in each group, the 25 th and 75 th percentile limits (Q1 and Q3) were calculated, the value of Q1 was subtracted from the value of Q3, the result was multiplied by 1.5, and the product was subtracted from Q1 and added to Q3 to modify the limits (Q1' and Q3'). Values under Q1' and over Q3' were removed.

As the Shapiro-Wilk normality test was not fulfilled in all groups and all parameters, Mann-Whitney U test was performed for hypothesis testing. Our results are presented in Figure 9-12. as boxplots (created in SigmaPlot 12.0 for Windows) indicated the median values with the $25^{\text {th }}$ and $75^{\text {th }}$ percentiles for all variables in all groups in order to be easily comparable to each other. 


\section{Results}

Altogether 27 child migraineurs, 27 healthy child controls, 22 adults migraineurs and 22 healthy adult volunteers participated in the visual guided equivalent learning test. 24 migraineurs children and 24 control children's data were analysed in the auditory paradigm, and 22-22 children data were analysed in the multisensory test.

\section{Data cleaning}

In the visual paradigm, altogether 18 entries were removed of the 392 (4.5\%), as follows: 3 entries were removed from NAT in the adult migraineurs group, 4 entries were removed from GER and 1 from RER in the adult controls group, 3 entries were removed from NAT, 1 from AER and 2 from RER in child migraineurs group, 4 entries were removed from NAT in child controls group (Table 2 and Table 3 ). 


\begin{tabular}{|c|c|c|c|c|c|c|c|}
\hline \multicolumn{4}{|c|}{ Adult migrainuers } & \multicolumn{4}{|c|}{ Adult controls } \\
\hline NAT & ALER & RER & GER & NAT & ALER & RER & GER \\
\hline 57 & 0,088 & 0,056 & 0,333 & 39 & 0,000 & 0,028 & 0,083 \\
\hline 120 & 0,217 & 0,083 & 0,667 & 71 & 0,085 & 0,083 & 0,250 \\
\hline 333 & 0,387 & 0,194 & 1,00 & 51 & 0,078 & 0,000 & 0,083 \\
\hline 91 & 0,220 & 0,000 & 0,083 & 58 & 0,103 & 0,028 & 0,250 \\
\hline 58 & 0,103 & 0,306 & 0,500 & 64 & 0,172 & 0,111 & 0,000 \\
\hline 60 & 0,083 & 0,028 & 0,000 & 41 & 0,024 & 0,028 & 0,000 \\
\hline 125 & 0,216 & 0,000 & 0,000 & 49 & 0,061 & 0,000 & 0,417 \\
\hline 75 & 0,107 & 0,000 & 1 & 78 & 0,128 & 0,000 & 0,000 \\
\hline 50 & 0,040 & 0,000 & 0,167 & 57 & 0,105 & 0,056 & 0,000 \\
\hline 52 & 0,077 & 0,056 & 0,500 & 76 & 0,132 & 0,028 & 0,083 \\
\hline 228 & 0,289 & 0,278 & 0,667 & 101 & 0,178 & 0,083 & 0,000 \\
\hline 45 & 0,044 & 0,000 & 0,500 & 47 & 0,021 & 0,028 & 0,083 \\
\hline 80 & 0,125 & 0,167 & 0,667 & 41 & 0,024 & 0,000 & 0,000 \\
\hline 89 & 0,303 & 0,000 & 0,00 & 45 & 0,044 & 0,000 & 0,000 \\
\hline 85 & 0,129 & 0,222 & 0,583 & 40 & 0,025 & 0,000 & 0,000 \\
\hline 93 & 0,183 & 0,139 & 0,667 & 60 & 0,083 & 0,056 & 0,083 \\
\hline 424 & 0,290 & 0,028 & 0,500 & 76 & 0,132 & 0,028 & 0,083 \\
\hline 73 & 0,110 & 0,111 & 1,000 & 75 & 0,133 & 0,056 & 0,000 \\
\hline 45 & 0,022 & 0,000 & 0,000 & 71 & 0,108 & 0,278 & 0,917 \\
\hline 42 & 0,048 & 0,000 & 1,000 & 66 & 0,182 & 0,056 & 0,000 \\
\hline 48 & 0,063 & 0,028 & 0,000 & 42 & 0,024 & 0,000 & 0,000 \\
\hline 340 & 0,376 & 0,000 & 0,583 & 67 & 0,090 & 0,000 & 0,083 \\
\hline
\end{tabular}

Table 2. The performances in the visually guided associative learning paradigm in adult population. Extreme outlayers are marked with different colours. NAT: number of acquisition trials; AER: association error ratio; RER: retrieval error ratio; GER: generalization error ratio. 


\begin{tabular}{|c|c|c|c|c|c|c|c|}
\hline \multicolumn{4}{|c|}{ Child migraineurs } & \multicolumn{4}{|c|}{ Child controls } \\
\hline NAT & AER & RER & GER & NAT & AER & RER & GER \\
\hline 69 & 0,087 & 0,167 & 0,833 & 50 & 0,060 & 0,056 & 0,000 \\
\hline 44 & 0,023 & 0,000 & 0,083 & 47 & 0,000 & 0,028 & 0,083 \\
\hline 45 & 0,000 & 0,139 & 0,083 & 74 & 0,095 & 0,000 & 0,083 \\
\hline 79 & 0,152 & 0,361 & 1,000 & 119 & 0,193 & 0,250 & 0,750 \\
\hline 59 & 0,068 & 0,000 & 0,083 & 67 & 0,090 & 0,139 & 0,167 \\
\hline 70 & 0,114 & 0,278 & 0,833 & 51 & 0,020 & 0,083 & 0,000 \\
\hline 137 & 0,175 & 0,028 & 0,083 & 60 & 0,083 & 0,083 & 0,083 \\
\hline 58 & 0,086 & 0,028 & 0,000 & 84 & 0,143 & 0,056 & 0,167 \\
\hline 59 & 0,085 & 0,083 & 0,167 & 58 & 0,086 & 0,000 & 0,000 \\
\hline 255 & 0,294 & 0,083 & 0,500 & 59 & 0,068 & 0,083 & 0,083 \\
\hline 56 & 0,089 & 0,000 & 0,250 & 57 & 0,088 & 0,056 & 0,083 \\
\hline 61 & 0,082 & 0,000 & 0,083 & 117 & 0,103 & 0,056 & 0,083 \\
\hline 45 & 0,000 & 0,000 & 0,083 & 136 & 0,154 & 0,028 & 0,083 \\
\hline 47 & 0,00 & 0,056 & 0,083 & 60 & 0,083 & 0,056 & 0,167 \\
\hline 60 & 0,050 & 0,083 & 0,417 & 69 & 0,072 & 0,056 & 0,250 \\
\hline 57 & 0,035 & 0,056 & 0,083 & 74 & 0,176 & 0,000 & 0,000 \\
\hline 55 & 0,073 & 0,028 & 0,083 & 124 & 0,185 & 0,167 & 0,500 \\
\hline 42 & 0,000 & 0,000 & 0,000 & 45 & 0,022 & 0,056 & 0,000 \\
\hline 42 & 0,000 & 0,056 & 0,000 & 48 & 0,021 & 0,028 & 0,000 \\
\hline 63 & 0,111 & 0,111 & 0,250 & 81 & 0,099 & 0,028 & 0,083 \\
\hline 122 & 0,148 & 0,139 & 0,667 & 52 & 0,019 & 0,056 & 0,083 \\
\hline 42 & 0,000 & 0,000 & 0,000 & 58 & 0,069 & 0,056 & 1,000 \\
\hline 55 & 0,091 & 0,028 & 0,167 & 82 & 0,098 & 0,028 & 0,417 \\
\hline 53 & 0,057 & 0,056 & 0,667 & 49 & 0,020 & 0,028 & 0,83 \\
\hline 68 & 0,088 & 0,000 & 0,000 & 41 & 0,000 & 0,000 & 0,083 \\
\hline 58 & 0,034 & 0,028 & 0,167 & 74 & 0,122 & 0,111 & 0,250 \\
\hline 79 & 0,114 & 0,167 & 1,000 & 52 & 0,058 & 0,000 & 0,250 \\
\hline
\end{tabular}

Table 3. The performances in the visually guided associative learning paradigm in child population. Extreme outlayers are marked with different colours. NAT: number of acquisition trials; AER: association error ratio; RER: retrieval error ratio; GER: generalization error ratio. 
In the auditory paradigm, altogether 8 entries were removed of the $192(4.2 \%)$, as follows: 1 entry was removed from AER and 1 entry from RER in the child migrianeurs group and 2-2 entry were removed from NAT, AER and RER in the child controls group (Table 4).

\begin{tabular}{|c|c|c|c|c|c|c|c|}
\hline \multicolumn{4}{|c|}{ Child migraineurs } & \multicolumn{4}{|c|}{ Child controls } \\
\hline NAT & AER & RER & GER & NAT & AER & RER & GER \\
\hline 79 & 0,051 & 0,028 & 0,333 & 49 & 0,020 & 0,000 & 0,000 \\
\hline 38 & 0,000 & 0,000 & 1,000 & 176 & 0,261 & 0,000 & 0,000 \\
\hline 40 & 0,000 & 0,000 & 0,083 & 47 & 0,021 & 0,000 & 0,000 \\
\hline 116 & 0,147 & 0,472 & 0,667 & 41 & 0,000 & 0,000 & 0,583 \\
\hline 46 & 0,022 & 0,028 & 0,000 & 112 & 0,188 & 0,194 & 0,167 \\
\hline 80 & 0,088 & 0,056 & 0,000 & 54 & 0,037 & 0,167 & 0,500 \\
\hline 44 & 0,000 & 0,056 & 0,000 & 41 & 0,000 & 0,111 & 0,250 \\
\hline 61 & 0,098 & 0,056 & 0,167 & 48 & 0,021 & 0,000 & 0,000 \\
\hline 59 & 0,068 & 0,111 & 0,750 & 48 & 0,000 & 0,000 & 0,083 \\
\hline 54 & 0,037 & 0,111 & 0,000 & 56 & 0,018 & 0,000 & 0,000 \\
\hline 46 & 0,022 & 0,000 & 0,000 & 54 & 0,037 & 0,028 & 0,250 \\
\hline 105 & 0,133 & 0,028 & 0,000 & 38 & 0,000 & 0,028 & 0,083 \\
\hline 47 & 0,021 & 0,111 & 0,500 & 50 & 0,000 & 0,056 & 0,417 \\
\hline 43 & 0,000 & 0,194 & 0,500 & 45 & 0,022 & 0,056 & 0,000 \\
\hline 52 & 0,038 & 0,028 & 0,000 & 62 & 0,048 & 0,056 & 0,417 \\
\hline 43 & 0,000 & 0,111 & 0,417 & 45 & 0,044 & 0,111 & 0,333 \\
\hline 49 & 0,102 & 0,083 & 0,250 & 51 & 0,020 & 0,000 & 0,083 \\
\hline 84 & 0,095 & 0,167 & 0,500 & 44 & 0,000 & 0,028 & 0,000 \\
\hline 42 & 0,000 & 0,167 & 0,417 & 64 & 0,047 & 0,028 & 1,000 \\
\hline 45 & 0,000 & 0,167 & 0,333 & 41 & 0,024 & 0,056 & 0,083 \\
\hline 68 & 0,059 & 0,278 & 0,583 & 50 & 0,040 & 0,028 & 0,083 \\
\hline 92 & 0,087 & 0,028 & 0,000 & 52 & 0,019 & 0,139 & 0,833 \\
\hline 49 & 0,020 & 0,000 & 0,250 & 43 & 0,000 & 0,056 & 0,500 \\
\hline 121 & 0,281 & 0,028 & 0,333 & 42 & 0,000 & 0,056 & 0,833 \\
\hline
\end{tabular}

Table 4. The performances in the auditory guided associative learning paradigm. Extreme outlayers are marked with different colours. NAT: number of acquisition trials; AER: association error ratio; RER: retrieval error ratio; GER: generalization error ratio. 
In the audiovisual paradigm, altogether 10 entries were removed of the $176(5.7 \%)$, as follows: 2 entries were removed from RER in the child migrianeurs group and 1-1 entry was removed from NAT and RER and 2 entries were removed from the AER and 3 entries were removed from GER in the child control (Table 5).

\begin{tabular}{|c|c|c|c|c|c|c|c|}
\hline \multicolumn{4}{|c|}{ Child migraineurs } & \multicolumn{4}{|c|}{ Child controls } \\
\hline NAT & RER & RER & GER & NAT & RER & RER & GER \\
\hline 70 & 0,100 & 0,083 & 0,000 & 66 & 0,076 & 0,000 & 0,000 \\
\hline 60 & 0,050 & 0,000 & 0,333 & 47 & 0,043 & 0,000 & 0,000 \\
\hline 53 & 0,038 & 0,056 & 0,167 & 46 & 0,000 & 0,000 & 0,000 \\
\hline 47 & 0,064 & 0,028 & 0,000 & 43 & 0,023 & 0,000 & 0,083 \\
\hline 58 & 0,069 & 0,083 & 0,333 & 163 & 0,209 & 0,278 & 0,583 \\
\hline 61 & 0,066 & 0,083 & 0,250 & 59 & 0,068 & 0,083 & 0,000 \\
\hline 60 & 0,067 & 0,361 & 0,333 & 53 & 0,038 & 0,056 & 0,000 \\
\hline 51 & 0,039 & 0,028 & 0,083 & 50 & 0,040 & 0,000 & 0,333 \\
\hline 48 & 0,042 & 0,000 & 0,000 & 41 & 0,000 & 0,000 & 0,000 \\
\hline 49 & 0,020 & 0,000 & 0,000 & 47 & 0,021 & 0,194 & 0,667 \\
\hline 49 & 0,020 & 0,000 & 0,000 & 53 & 0,038 & 0,028 & 0,167 \\
\hline 43 & 0,023 & 0,000 & 0,000 & 42 & 0,024 & 0,000 & 0,083 \\
\hline 52 & 0,058 & 0,056 & 0,417 & 51 & 0,059 & 0,000 & 0,000 \\
\hline 75 & 0,080 & 0,056 & 0,333 & 76 & 0,118 & 0,111 & 0,250 \\
\hline 58 & 0,069 & 0,167 & 0,417 & 72 & 0,139 & 0,083 & 0,000 \\
\hline 80 & 0,063 & 0,028 & 0,083 & 55 & 0,127 & 0,083 & 0,083 \\
\hline 64 & 0,094 & 0,611 & 0,667 & 49 & 0,061 & 0,000 & 0,083 \\
\hline 43 & 0,000 & 0,000 & 0,000 & 48 & 0,021 & 0,028 & 0,000 \\
\hline 45 & 0,044 & 0,028 & 0,167 & 41 & 0,000 & 0,028 & 0,000 \\
\hline 64 & 0,063 & 0,000 & 0,250 & 61 & 0,066 & 0,028 & 0,000 \\
\hline 54 & 0,056 & 0,000 & 0,083 & 61 & 0,049 & 0,028 & 0,000 \\
\hline 41 & 0,000 & 0,000 & 0,333 & 42 & 0,024 & 0,000 & 0,000 \\
\hline
\end{tabular}

Table 5. The performances in the multisensory guided associative learning paradigm. Extreme outlayers are marked with different colours. NAT: number of acquisition trials; AER: acquisition error ratio; RER: retrieval error ratio; GER: generalization error ratio. 


\section{Results of the visually guided associative learning paradigm}

The child migraineur and the child control groups did not differ significantly regarding the median number of the trials required for completing the acquisition phase (58 $(\min =42, \max =255)$ vs $60(\min =41 ; \max =136) ; \mathrm{U}=235.5, p=0.395)$. The median error ratios during the acquisition phase was similar in the two investigated child groups $(0.08$ ( $\mathrm{min}=0.00$, $\max =0.29)$ vs. $0.09(\min =0.00, \max =0.19) ; \mathrm{U}=300.0, p=0.369)$. Statistical analysis of the median error ratios in the retrieval part of the test phase did not show significant difference between the children migraneurs and the children control populations $(0.06$ ( $\mathrm{min}=0.00$, $\max =0.36)$ vs. $0.06(\min =0.000, \max =0.25) ; \mathrm{U}=310.0, p=0.621)$. There was also no significant difference between children patients and controls in terms of the median error ratios in the generalization part of the test phase $(0.08$ ( $\min =0.00, \max =1.00)$ vs. 0.08 $(\min =0.00, \max =1.00) ; \mathrm{U}=323.5, p=0.484)$ (Figure 9). 
A

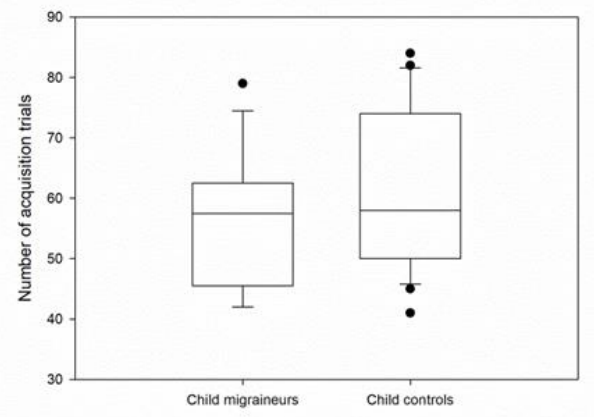

C

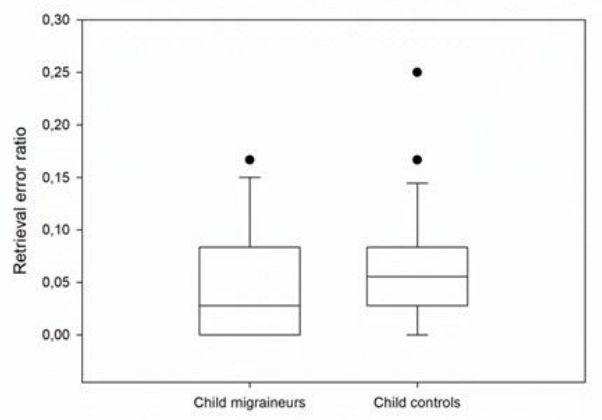

B

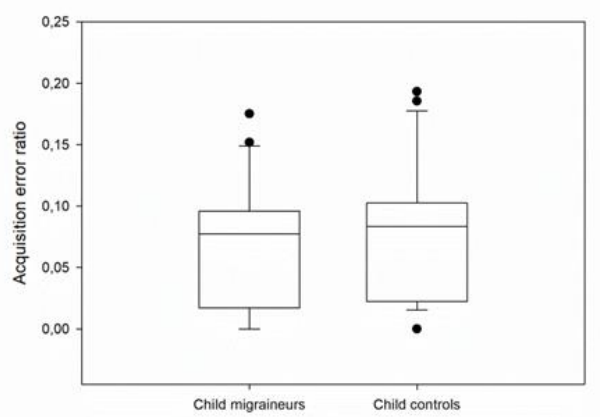

D

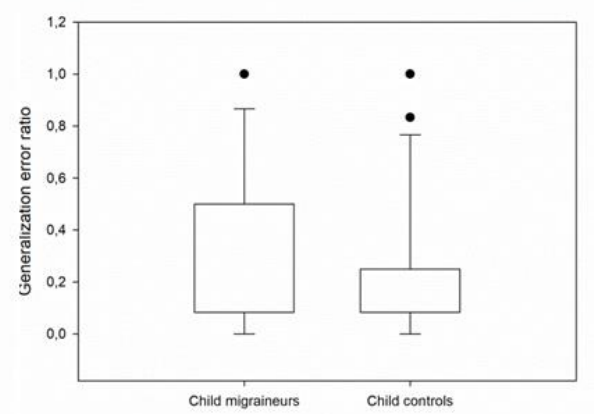

Figure 9. Performance on the visually guided associative learning test in children groups: the number of trials required for completing the acquisition phase (A), the ratio of the correct and incorrect answers in the acquisition phase (B), retrieval (C) and generalization (D) parts of the test phase in the child migraineur and control groups. The lower margin of the boxes shows the $25^{\text {th }}$ percentile, the line within the boxes marks the median, and the upper margin of the boxes indicates the $75^{\text {th }}$ percentile. The error bars (whiskers) above and below the boxes indicates the $90^{\text {th }}$ and $10^{\text {th }}$ percentiles. The dots represent the outliers. 
In the adult migraineur group the median number of the trials required for completing the acquisition phase was not significantly higher than in the adult control population $(77(\min =42, \max =424)$ vs. $59(\min =39 ; \max =101) ; \mathrm{U}=141.5, p=0.08)$. The median error ratio during the acquisition phase was significantly increased in the adult migraineur group as compared to the adult control group $(0.12(\min =0.02, \max =0.39)$ vs. 0.09 ( $\min =0.000, \max =0.18) ; \mathrm{U}=155.5, p=0.043)$. The median error ratios during the retrieval part of the test phase were similar in the adult migraineur and adult control groups/adult cases vs. controls $(0.03(\min =0.000, \max =0.31)$ vs. $0.03(\min =0.000, \max =0.28) ; \mathrm{U}=195.5, p=0.395)$. But a significantly higher median error ratio in the generalization part of the test phase was found among the adult patients as compared to the adult controls $(0.50(\min =0.00, \max =1.00)$ vs. $0.04(\min =0.00, \max =0.92) ; \mathrm{U}=66.0, p<0.001)$ (Figure 10). 
A

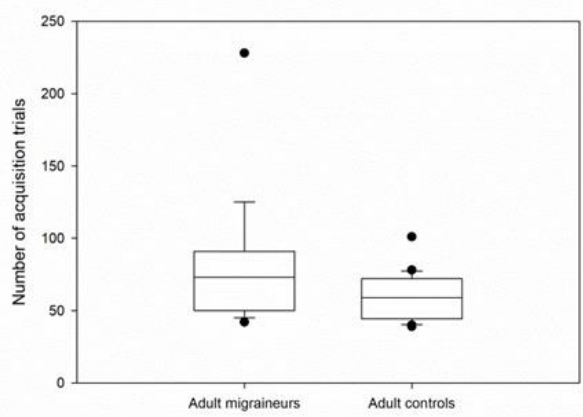

C

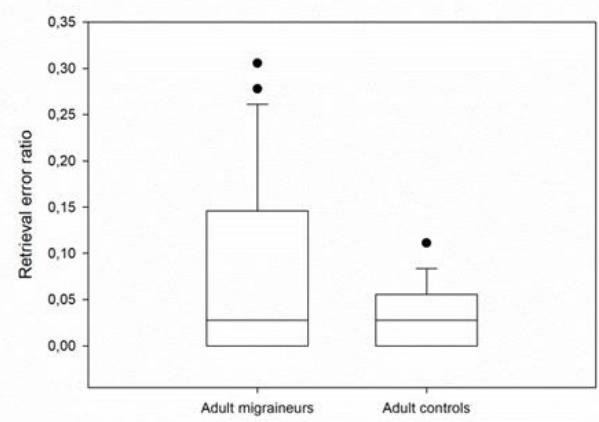

B

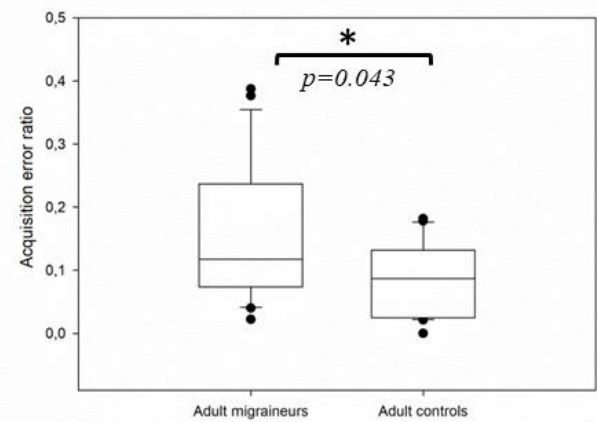

D

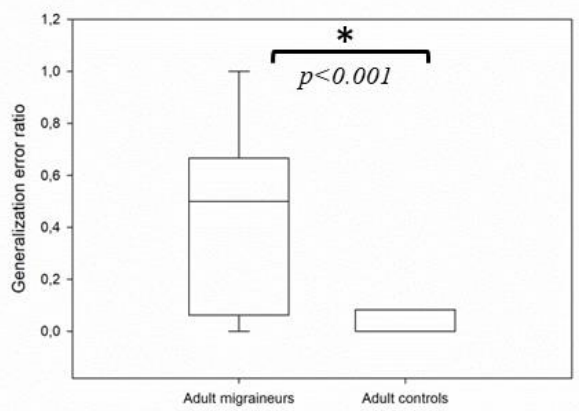

Figure 10. Performance on the visually guided associative learning test in adult groups: the number of trials required for completing the acquisition phase (A), the ratio of the correct and incorrect answers in the acquisition phase (B), retrieval (C) and generalization (D) parts of the test phase in the adult migraineur and control groups. The lower margin of the boxes shows the 25th percentile, the line within the boxes marks the median, and the upper margin of the boxes indicates the 75th percentile. The error bars (whiskers) above and below the boxes indicates the 90th and 10th percentiles. The dots represent the outliers. 


\section{Results of auditory equivalence learning paradigm}

The child migraineur and the child control groups did not differ significantly regarding the median number of the trials required for completing the acquisition phase $(50.5$ $(\min =38, \max =121)$ vs $48 \quad(\min =38 ; \max =48) ; \mathrm{U}=193.5, p=0.123)$. There was not significantly higher among the child patients as compared to the children controls in term of the median error ratios in the acquisition phase $(0.04 \quad(\min =0.00, \max =0.15)$ vs. 0.02 ( $\min =0.00, \max =0.05) ; \mathrm{U}=170, p=0.06$ ). The median error ratios in the retrieval part of the test phase did not differ significantly between the two investigated child groups (0.06 $(\min =0.000, \max =0.28)$ vs. $0.03(\min =0.00, \max =0.14) ; \mathrm{U}=170, p=0.06)$. Statistical analysis of the median error ratios in the generalization part of the test phase did not show significant difference between the child migraneur and the child control populations $(0.29$ ( $\min =0.00$, $\max =1.00)$ vs. $0.13(\min =0.00, \max =1.00) ; \mathrm{U}=274.5, p=0.79)$ (Figure 11). 
A

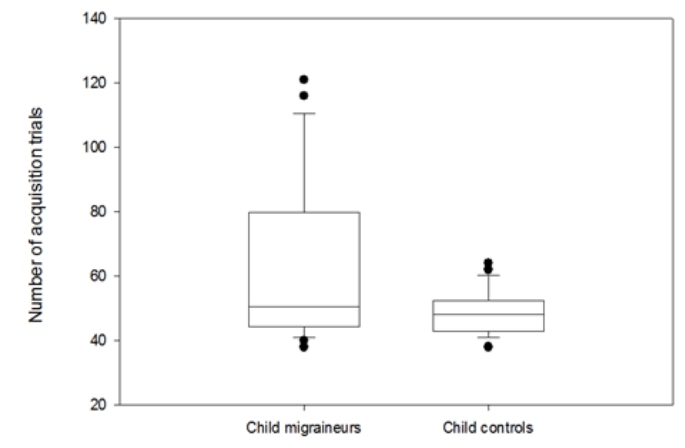

C

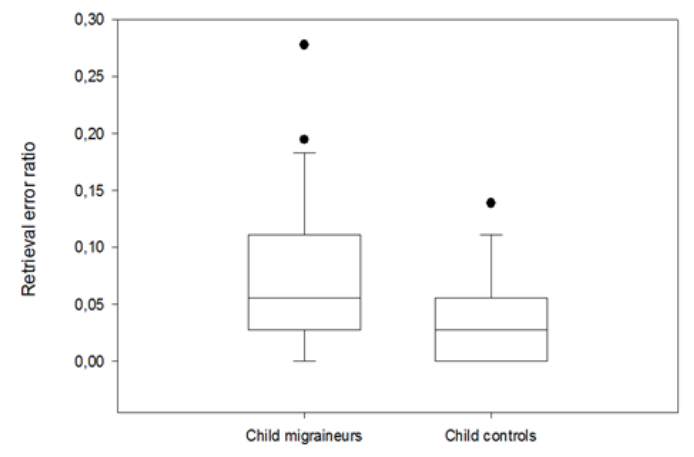

\section{B}

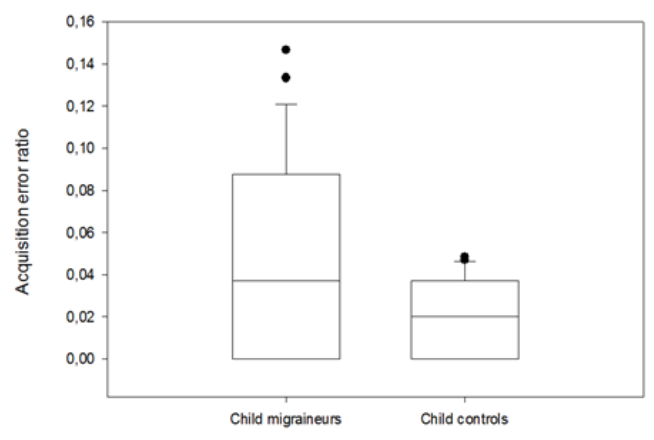

D

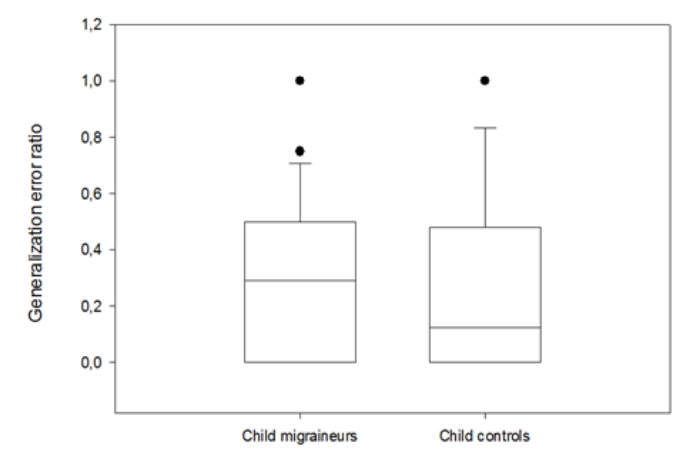

Figure 11. Performance on the auditory equivalent learning test: the number of trials required for completing the acquisition phase (A), the ratio of the correct and incorrect answers in the acquisition phase (B), retrieval (C) and generalization (D) parts of the test phase in the adult and child control groups. The lower margin of the boxes shows the 25 th percentile, the line within the boxes marks the median, and the upper margin of the boxes indicates the 75th percentile. The error bars (whiskers) above and below the boxes indicates the 90th and 10th percentiles. The dots represent the outliers. 


\section{Results of audiovisual equivalence learning paradigm}

The median number of the trials needed to complete the acquisition phase was similar between child migraineurs and child controls $(53.5 \quad(\min =41 ; \max =80)$ vs. $50 \quad(\min =41$, $\max =76) ;(\mathrm{U}=187, p=0.29)$. There was also no significant difference between the two investigated control groups in term of the mean error ratios in the acquisition phase $(0.06$ $(\min =0.00, \max =0.1)$ vs. $(0.04(\min =0.00, \max =0.13) ; \mathrm{U}=180, p=0.32)$. The median error ratios during the retrieval part of the test phase were similar in the child cases compared to controls $(0.03$ ( $\min =0.00, \max =0.17)$ vs. (0.03 ( $\min =0.00, \max =0.19) ; \mathrm{U}=198.5, p=0.98)$. However, a significantly higher median error ratio in the generalization part of the test phase was found among the child patients as compared to the child controls $(0.17$ ( $\mathrm{min}=0.000$, $\max =0.67)$ vs. $0.00(\min =0.00, \max =0.17) ; \mathrm{U}=90.5, p=0.002)($ Figure 12). 

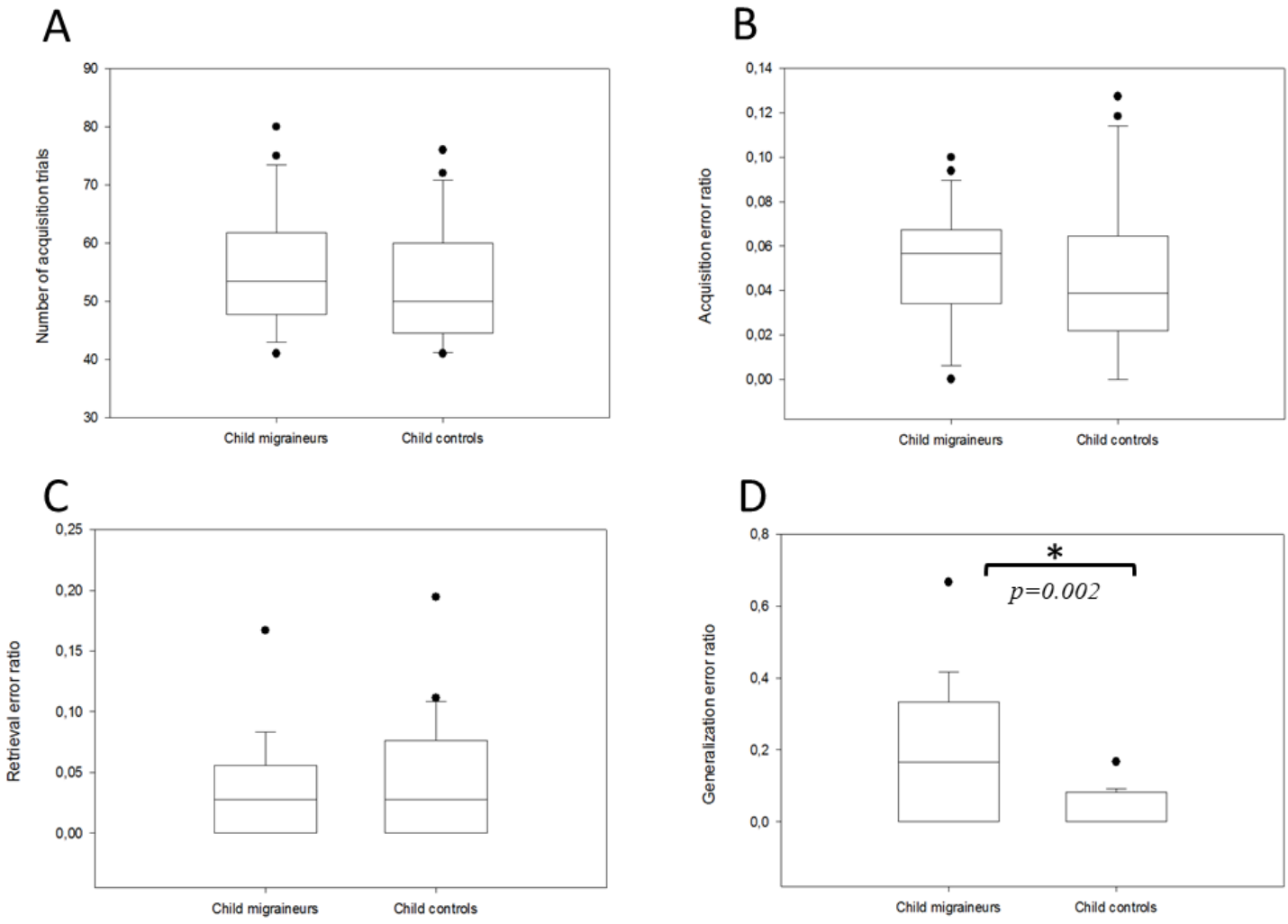

Figure 12. Performance on the multisensory equivalent learning test: the number of trials required for completing the acquisition phase (A), the ratio of the correct and incorrect answers in the acquisition phase (B), retrieval (C) and generalization (D) parts of the test phase in the adult and child control groups. The lower margin of the boxes shows the 25th percentile, the line within the boxes marks the median, and the upper margin of the boxes indicates the 75th percentile. The error bars (whiskers) above and below the boxes indicates the 90th and 10th percentiles. The dots represent the outliers. 


\section{Discussion}

The visually-guided RAET [3] was originally developed to explore the visually guided associative learning of neurological patients with basal ganglia and hippocampal dysfunction. The paradigm consists of two main parts. In the acquisition part, the participants must learn to associate pairs of visual stimuli and the equivalence testing or transfer part includes new, hitherto not presented pairings of the same stimuli. If the functional equivalence has been successfully established, the participants will have no difficulty with these new pairings. The association of new stimuli is dominated by the function of the basal ganglia and the coding and recall of associations are mainly a function of the medial temporal lobe (including hippocampi). The test was later used in psychiatric disorders [10] as well as in electroencephalogram (EEG) study of healthy subjects, too [129]. A recent study of our research group revealed impairment of this associative learning function in adult migraineurs [124]. The main novelty of the presented thesis work is the description of the effect of the childhood migraine on this function. To our knowledge, this is the first systematic study, which addresses the sensory guided associative learning in pediatric migraine patients. Our results revealed in contrast to the adult migrainours that the associative learning functions are not altered in the children patient population.

Migraine is a primary headache, one of the most prevalent chronic neurological disorders with extremely incapacitating symptoms affecting about 1.04 billion people worldwide that correspond to a prevalence of $11 \%[33,34]$. Migraine without aura is characterized by recurrent headaches manifesting in attacks lasting 4-72 hours typically accompained by at least two of the following symptoms: unilateral location, pulsating quality, aggravation by routine physical activity. Further associated migraine symptoms include nausea, vomiting, phonophobia, and photophobia [31]. The manifestation of migraine may also be connected to children's lifestyle, psychological stress, excessive physical fatigue and exposure to strong light effects [50]. Cognitive and memory functions in child migraineurs, as well as the visuo-cognitive processing have been found not to be working properly in patients suffering from migraine, both with and without aura [120-122]. We have performed a series of studies on contour integration, dynamic contrast sensitivity and motion coherence processing in order to make exploratory observations about the changes in visual perception in children and adolescents suffering from migraine. We found that migraine-suffering children have slower developing visual contour integration and reduced motion coherence 
processing capacity. Furthermore the contrast sensitivity is mostly affected at low spatial frequencies in child migraineurs [117-119].

The present thesis tested a simple hypothesis. We hypothesized that similarly to the adult migraineours the pediatric patient population would show significantly poorer performance in at least one phase of our visual learning paradigms than age- and sex-matched controls. In other words, we hypothesized that deficits observed in adults [124] would be observable already in the pediatric population, indicating some sort of inherent structural or functional alteration of the underlying neural structures. The discussion is confined to this hypothesis, especially as we do not wish to deal with questions that have been addressed in other studies better designed to answer those specific questions. We also investigated a cohort of 265 healthy subjects with an age range of 3-52 years how performance in different tasks of the RAET changes with age and found a significant effect on associative learning and on retrival of the learned pairs, but not on generalization [130].

The current study failed to support the hypothesis. The difference between the pediatric groups was not significant in any of the studied parameters in the visual paradigm. However, we must mention limitations of our experiments. This study included the relatively low number of participants (due to and partially offset by the strict application of the diagnostic criteria). Although, in our opinion, these limitations do not affect the validity of the conclusion that the equivalence learning deficit observed in adult migraine patients does not exist in childhood.

In contrast, as already discussed in our previous study [124], adult patients show significantly lower performance in both acquisition and generalization than matched controls: adult migraineurs acquired visual stimulus pairings and the pairing rule with greater difficulty than matched controls, but having acquired them, their recall performance for already seen stimulus pairs was on par with that of controls. When it came to generalizing the pairing rule, though, they performed significantly worse. To put it simply, no difference in childhood turns into a large gap by adulthood, and this is especially true for generalization. The question logically arises: what happens in the meantime? This is a question only a large longitudinal study supported by instrumental measurements could exactly answer. The available literature offers some clues, though. An increasing number of studies come to the conclusion that migraine affects both areas that are of key importance for the successful completion of the studied task: the BG and the hippocampi $[100,131,132]$. One interesting study would be the direct comparison of the performances of adult and children patient groups in the visually 
guided associative learning paradigm. This could provide a more complete picture about the effect of migraine in this kind of learning. However, this require data from more participants. This is the reason that we did not stop at this point the experimental work and we continue the collecting of behavioral data of further children and adult migraineurs.

In a previous fMRI study, it has been found that migraine has a negative effect on the proper functioning of the $\mathrm{BG}$ and that this damage is proportional to the frequency of attacks [100]. It was described in another study that volumes of the right globus pallidus, left globus pallidus, and left putamen were significantly smaller in migraine patients (with aura) than in healthy controls $[123,133]$. Another research group examined persons living with migraine without aura and concluded that the volume of the nucleus accumbens and caudate nucleus of patients was significantly smaller than that of controls [97].

As for the hippocampi, Maleki's group found significantly larger bilateral hippocampal volume in migraine patients with low attack frequency than in the highfrequency and control groups [101]. Liu and colleagues found that the frequency of headaches, number of attacks, anxiety and depression scores, and genetic effects contribute to functional changes in the hippocampus in migraine patients [134]. The same group also published that the hippocampus and amygdala displayed a structural plasticity linked to both headache frequency and clinical outcome of migraine [134].

Concerning the auditory guided associative learning, we can conclude that there are no significant differences in the performances between the pediatric patient and control groups. Thus, the performances of the pediatric migraine patients similarly to those of the visually associative learning are also not altered here. We have to mention here that the auditory guided task does not totally correspond to the visual and multisensory guided ones. Although all of the learning tasks contain eight stimuli, in the auditory paradigm in contrast to the visual and multisensory test where two visual or an auditory and a visual stimuli had to be associated the sound has to be associated not to a second sound but to a particular button on the keyboard. In an earlier draft of the auditory paradigm we tried to apply two different sounds in the two ears but the participants would quickly become nervous and were not able to learn the acquisitions at all. However, the influence of this difference on the results cannot be explained by the auditory association to a keyboard button, as it seems to be an easier task than the visual and visual auditory associations, nevertheless the performances were worst in the auditory test [127]. 
Developmental studies tell us that the human striatum shows protracted development, well into the adolescence [135], while the hippocampi never really seem to finish development. Thus, both structures are vulnerable for a long period. In fact, it is safe to assume that the hippocampi - due to their extreme plasticity - remain sensitive to insults throughout one's life. The other side of the hippocampal plasticity coin is, of course, that dynamic plasticity offers a way to remodel and regenerate once the insult is gone. Unfortunately, no study has ever examined this question in the context of migraine, but it would be intriguing to see if hippocampus-related cognitive functions improve after a prolonged attack-free period.

Recent results of our research group revealed no significant difference among the performance (error ration) in the unimodal visual, unimodal auditory and the combined audiovisual paradigms in the acquisition phase in healthy humans [127]. Similarly, we have found no differences between the equivalence learning of pediatric patients and control children irrespectively of the stimulus modality. Thus, the modality of the stimuli doesn't affect the performance in this phase of the behavioral test. Thus the feedback based pair learning a very old and conserved function which can be linked to an ancient structure, the basal ganglia is not affected by pediatric migraine. In contrast, the generalization part of the test phase, which is modality dependent [127] seems to be affected in pediatric patients. The only significant difference, which was found in the comparison between the pediatric patients and healthy controls in our research, was in the genaralization error ratio of the multisensory test. The generalization in the audio-visual paradigm is the most complicated part of the applied three paradigms and if there are any alteration in the sensory guided associative learning it is the easiest to detect here because of its complexity. Multimodal facilitation plays a role in visual perception [136] object recognition [137, 138] emotional changes recognition [139], face and voice recognition [140], or in person recognition [141]. This interesting finding could shed light on the altered multisensory integration even in pediatric migraine patients, which can be elicit a significant decrease in the performance of the generalization part of the multisensory paradigm. 


\section{Conclusion}

Our results demonstrated no significant deficit of equivalence learning and the connected memory processes in pediatric migraine patients in visual and auditory learning paradigms and in the acquisition and the retrieval parts of the multisensory paradigm. Our results suggest that the loss of the visual associative learning function in adult patients is not an inherent feature of the migrainous cognitive profile rather the result of the attacks 'interference with the development / function of the underlying structures. The only significant deficit in the performance of pediatric patients was in the generalization part of the multisensory learning paradigm. The altered generalization of multisensory stimuli because of most probably from the altered multisensory integration could be an early signal of the loss of associative learning function in children and adolescent patient suffering in migraine. 


\section{Acknowledgements}

First of all, I would like to thank my supervisor Dr. Attila Nagy, who guided my path through three years of scientific students' research, thesis and $\mathrm{PhD}$ work to writing this dissertation.

I would also like to thank my gratitude to Professor Gábor Jancsó and Professor Ferenc Bari for giving me the chance to participate in the Neuroscience $\mathrm{PhD}$ program and $\mathrm{I}$ am also grateful to Professor Gyula Sáry for allowing me to do my research in the Department of Physiology.

I would like to thank Dr. Ágnes Fehér and Dr. András Puszta, who have always inspired me and always turned to me with patience and love. Thank you also for helping me so much with my scientific work. Their scientific approach and supportive advices they showed towards me is something that I would never forget.

I would like to thank Dr. Gábor Braunitzer and Dr. Gabriella Eördegh for their help with the migraine research, statistics and writing the article.

I am also grateful to Dr. Balázs Bodosi for the great help in setting up the experimental software and to Péter Liszli for the informatics background.

A deep thank you goes out to my fellow PhD students, namely Dr. Attila Öze, Dr. Ákos Pertich, Diána Nyujtó, Viktória Balikó, Xénia Katona and postdoc Dr. Balázs Barkóczi. I would not have had such beautiful years without you, the many common experiences we had together will stay with me forever. I would also like to thank all the insipration and that comes from our stundents, András Hegedűs, Dr. Anna Pihokker, Dr. Blanka Bindics, Dr. Dóra Dózsai, Márton Edelmayer, Mercédesz Kovács, Nándor Görög and Dr. Nóra Cserháti.

I would like to express my deepest thanks to all the collegues of the Department of Physiology, who have always been kind and made my daily life better, specially to Dr. Alexandra Büki, Dr. András Benyhe, Dr. Péter Kaposvári and Dr. Zoltán Lelkes.

I would like to thank to neurologists Dr. Jenő Kóbor, Dr. Katalin Bihari and Dr. Éva Pálinkás, who sent us the carefully diagnostized pediatric and adult migraine patients.

I couldn't emphasize the gratitude towards my family, my mother, Andrea Jancsik, my stepfather, Péter Ábrahám, my grandparents, Emese Megyeri, Margit Gligor, Sándor Giricz ${ }^{\dagger}$ and Zoltán Jancsik, and all the members of my family. Thank you for always standing by me, I can always count on your help and for being your family. Without you, it would not have been possible.

I would also like to thank my partner, Bálint Szabados-Tóth, who accompanied me along this journey, took my hand when needed and always inspired me with his art. 
I am very grateful to my friends, who have always listened and encouraged me, particularly Dr. Ágota Kopniczky, Dorottya Ottlik, Fanni Czirok, Linda Vecseri and Dr. Zita Timár, whom I can always count on since our high school years. And finally, I would like to thank everyone who inspired or helped me in any way: colleagues in the Department of Physiology, in the Department of Psychiatric and in the Department of Physiology, Anatomy and Neuroscience, who taught me a lot; to the artists who influenced me and my thinking; the people I've met in my life, even for just a minute, but they've shown how wonderful life is.

The study was supported by the University of Szeged Grant SZTE-ÁOK-KKA (Grant No. 2019/270-62-2.) 


\section{References}

1. Delamater, A.R. and K.M. Lattal, The study of associative learning: mapping from psychological to neural levels of analysis. Neurobiol Learn Mem, 2014. 108: p. 1-4.

2. Haselgrove, M. and I. McLaren, The psychology of associative learning: Editorial. Q J Exp Psychol (Hove), 2019. 72(2): p. 93-97.

3. Myers, C.E., et al., Dissociating hippocampal versus basal ganglia contributions to learning and transfer. J Cogn Neurosci, 2003. 15(2): p. 185-93.

4. Meeter, M., D. Shohamy, and C.E. Myers, Acquired equivalence changes stimulus representations. J Exp Anal Behav, 2009. 91(1): p. 127-41.

5. Squire, L.R., Memory systems of the brain: a brief history and current perspective. Neurobiol Learn Mem, 2004. 82(3): p. 171-7.

6. Maddox, W.T. and J.V. Filoteo, Striatal contributions to category learning: quantitative modeling of simple linear and complex nonlinear rule learning in patients with Parkinson's disease. J Int Neuropsychol Soc, 2001. 7(6): p. 710-27.

7. Shohamy, D., et al., Role of the basal ganglia in category learning: how do patients with Parkinson's disease learn? Behav Neurosci, 2004. 118(4): p. 676-86.

8. Myers, C.E., et al., Hippocampal atrophy disrupts transfer generalization in nondemented elderly. J Geriatr Psychiatry Neurol, 2002. 15(2): p. 82-90.

9. Collie, A., et al., Selectively impaired associative learning in older people with cognitive decline. J Cogn Neurosci, 2002. 14(3): p. 484-92.

10. Keri, S., et al., Dissociation between medial temporal lobe and basal ganglia memory systems in schizophrenia. Schizophr Res, 2005. 77(2-3): p. 321-8.

11. Graybiel, A.M., The basal ganglia. Curr Biol, 2000. 10(14): p. R509-11.

12. Parent, A. and L.N. Hazrati, Functional anatomy of the basal ganglia. I. The corticobasal ganglia-thalamo-cortical loop. Brain Res Brain Res Rev, 1995. 20(1): p. 91127.

13. Parent, A. and L.N. Hazrati, Functional anatomy of the basal ganglia. II. The place of subthalamic nucleus and external pallidum in basal ganglia circuitry. Brain Res Brain Res Rev, 1995. 20(1): p. 128-54. 
14. McHaffie, J.G., et al., Subcortical loops through the basal ganglia. Trends Neurosci, 2005. 28(8): p. 401-7.

15. Shipp, S., The functional logic of corticostriatal connections. Brain Struct Funct, 2017. 222(2): p. 669-706.

16. Packard, M.G. and B.J. Knowlton, Learning and memory functions of the Basal Ganglia. Annu Rev Neurosci, 2002. 25: p. 563-93.

17. Albin, R.L., A.B. Young, and J.B. Penney, The functional anatomy of basal ganglia disorders. Trends Neurosci, 1989. 12(10): p. 366-75.

18. Worbe, Y., et al., Functional immaturity of cortico-basal ganglia networks in Gilles de la Tourette syndrome. Brain, 2012. 135(Pt 6): p. 1937-46.

19. Modell, J.G., et al., Neurophysiologic dysfunction in basal ganglia/limbic striatal and thalamocortical circuits as a pathogenetic mechanism of obsessive-compulsive disorder. J Neuropsychiatry Clin Neurosci, 1989. 1(1): p. 27-36.

20. Squire, L.R. and S. Zola-Morgan, The medial temporal lobe memory system. Science, 1991. 253(5026): p. 1380-6.

21. Squire, L.R., C.E. Stark, and R.E. Clark, The medial temporal lobe. Annu Rev Neurosci, 2004. 27: p. 279-306.

22. Corkin, S., et al., H. M.'s medial temporal lobe lesion: findings from magnetic resonance imaging. J Neurosci, 1997. 17(10): p. 3964-79.

23. Stefanacci, L., et al., Profound amnesia after damage to the medial temporal lobe: A neuroanatomical and neuropsychological profile of patient E. P. J Neurosci, 2000. 20(18): p. 7024-36.

24. Zola-Morgan, S., L.R. Squire, and S.J. Ramus, Severity of memory impairment in monkeys as a function of locus and extent of damage within the medial temporal lobe memory system. Hippocampus, 1994. 4(4): p. 483-95.

25. Brown, T.I. and C.E. Stern, Contributions of medial temporal lobe and striatal memory systems to learning and retrieving overlapping spatial memories. Cereb Cortex, 2014. 24(7): p. 1906-22. 
26. Voss, J.L., et al., Spontaneous revisitation during visual exploration as a link among strategic behavior, learning, and the hippocampus. Proc Natl Acad Sci U S A, 2011. 108(31): p. E402-9.

27. Keri, S., et al., Classification learning in Alzheimer's disease. Brain, 1999. 122 ( Pt 6): p. 1063-8.

28. Jeneson, A., K.N. Mauldin, and L.R. Squire, Intact working memory for relational information after medial temporal lobe damage. J Neurosci, 2010. 30(41): p. 13624-9.

29. Kirwan, C.B., et al., Detailed recollection of remote autobiographical memory after damage to the medial temporal lobe. Proc Natl Acad Sci U S A, 2008. 105(7): p. 2676-80.

30. Race, E., M.M. Keane, and M. Verfaellie, Medial temporal lobe damage causes deficits in episodic memory and episodic future thinking not attributable to deficits in narrative construction. J Neurosci, 2011. 31(28): p. 10262-9.

31. Headache Classification Committee of the International Headache, S., The International Classification of Headache Disorders, 3rd edition (beta version). Cephalalgia, 2013. 33(9): p. 629-808.

32. Olesen, J., Reliability of the nitroglycerin provocative test in the diagnosis of neurovascular headaches. Cephalalgia, 2005. 25(8): p. 636-7; author reply 637-9.

33. Stovner, L., et al., The global burden of headache: a documentation of headache prevalence and disability worldwide. Cephalalgia, 2007. 27(3): p. 193-210.

34. Lipton, R.B., et al., Migraine prevalence, disease burden, and the need for preventive therapy. Neurology, 2007. 68(5): p. 343-9.

35. Stewart, W.F., et al., Cumulative lifetime migraine incidence in women and men. Cephalalgia, 2008. 28(11): p. 1170-8.

36. Bank, J. and S. Marton, Hungarian migraine epidemiology. Headache, 2000. 40(2): p. 164-9.

37. Gesztelyi, G. and D. Bereczki, Primary headaches in an outpatient neurology headache clinic in East Hungary. Eur J Neurol, 2004. 11(6): p. 389-95.

38. Giffin, N.J., et al., Premonitory symptoms in migraine: an electronic diary study. Neurology, 2003. 60(6): p. 935-40. 
39. Rossi, P., A. Ambrosini, and M.G. Buzzi, Prodromes and predictors of migraine attack. Funct Neurol, 2005. 20(4): p. 185-91.

40. Silberstein, S.D. and W.B. Young, Migraine aura and prodrome. Semin Neurol, 1995. 15(2): p. 175-82.

41. Lauritzen, M., Pathophysiology of the migraine aura. The spreading depression theory. Brain, 1994. 117 ( Pt 1): p. 199-210.

42. Viana, M., et al., Clinical features of migraine aura: Results from a prospective diaryaided study. Cephalalgia, 2017. 37(10): p. 979-989.

43. Giffin, N.J., et al., The migraine postdrome: An electronic diary study. Neurology, 2016. 87(3): p. 309-13.

44. Blau, J.N., Migraine postdromes: symptoms after attacks. Cephalalgia, 1991. 11(5): p. 229-31.

45. Winner, P., et al., Classification of pediatric migraine: proposed revisions to the IHS criteria. Headache, 1995. 35(7): p. 407-10.

46. Raucci, U., et al., Management of Childhood Headache in the Emergency Department. Review of the Literature. Front Neurol, 2019. 10: p. 886.

47. Lewis, D.W., Pediatric migraine. Neurol Clin, 2009. 27(2): p. 481-501.

48. Hershey, A.D., Current approaches to the diagnosis and management of paediatric migraine. Lancet Neurol, 2010. 9(2): p. 190-204.

49. Merison, K. and H. Jacobs, Diagnosis and Treatment of Childhood Migraine. Curr Treat Options Neurol, 2016. 18(11): p. 48.

50. Ozge, A., et al., Overview of diagnosis and management of paediatric headache. Part I: diagnosis. J Headache Pain, 2011. 12(1): p. 13-23.

51. Barnes, N.P., Migraine headache in children. BMJ Clin Evid, 2015. 2015.

52. Gupta, S., et al., Potential role of female sex hormones in the pathophysiology of migraine. Pharmacol Ther, 2007. 113(2): p. 321-40.

53. Stewart, W.F., et al., Age- and sex-specific incidence rates of migraine with and without visual aura. Am J Epidemiol, 1991. 134(10): p. 1111-20. 
54. Teleanu, R.I., et al., Treatment of Pediatric Migraine: a Review. Maedica (Buchar), 2016. 11(2): p. 136-143.

55. Schreiber, C.P., et al., Prevalence of migraine in patients with a history of selfreported or physician-diagnosed "sinus" headache. Arch Intern Med, 2004. 164(16): p. 1769-72.

56. Vannatta, K., et al., Multiple perspectives on the psychological functioning of children with and without migraine. Headache, 2008. 48(7): p. 994-1004.

57. Pakalnis, A., et al., Serotonin effects on sleep and emotional disorders in adolescent migraine. Headache, 2009. 49(10): p. 1486-92.

58. Parsons, A.A. and P.J. Strijbos, The neuronal versus vascular hypothesis of migraine and cortical spreading depression. Curr Opin Pharmacol, 2003. 3(1): p. 73-7.

59. Goadsby, P.J. and L. Edvinsson, The trigeminovascular system and migraine: studies characterizing cerebrovascular and neuropeptide changes seen in humans and cats. Ann Neurol, 1993. 33(1): p. 48-56.

60. Edvinsson, L. and R. Uddman, Neurobiology in primary headaches. Brain Res Brain Res Rev, 2005. 48(3): p. 438-56.

61. Pietrobon, D. and M.A. Moskowitz, Pathophysiology of migraine. Annu Rev Physiol, 2013. 75: p. 365-91.

62. Borsook, D. and R. Burstein, The enigma of the dorsolateral pons as a migraine generator. Cephalalgia, 2012. 32(11): p. 803-12.

63. Weiller, C., et al., Brain stem activation in spontaneous human migraine attacks. Nat Med, 1995. 1(7): p. 658-60.

64. Akerman, S., P.R. Holland, and P.J. Goadsby, Diencephalic and brainstem mechanisms in migraine. Nat Rev Neurosci, 2011. 12(10): p. 570-84.

65. Goadsby, P.J., et al., Neurobiology of migraine. Neuroscience, 2009. 161(2): p. 32741.

66. May, A. and P.J. Goadsby, The trigeminovascular system in humans: pathophysiologic implications for primary headache syndromes of the neural influences on the cerebral circulation. J Cereb Blood Flow Metab, 1999. 19(2): p. 115-27. 
67. Olesen, J., et al., Origin of pain in migraine: evidence for peripheral sensitisation. Lancet Neurol, 2009. 8(7): p. 679-90.

68. Bernstein, C. and R. Burstein, Sensitization of the trigeminovascular pathway: perspective and implications to migraine pathophysiology. J Clin Neurol, 2012. 8(2): p. 89-99.

69. Fusco, M., et al., Neurogenic inflammation in primary headaches. Neurol Sci, 2003. 24 Suppl 2: p. S61-4.

70. Malhotra, R., Understanding migraine: Potential role of neurogenic inflammation. Ann Indian Acad Neurol, 2016. 19(2): p. 175-82.

71. Ramachandran, R., Neurogenic inflammation and its role in migraine. Semin Immunopathol, 2018. 40(3): p. 301-314.

72. Goadsby, P.J. and P.R. Holland, An Update: Pathophysiology of Migraine. Neurol Clin, 2019. 37(4): p. 651-671.

73. Stepien, A. and M. Chalimoniuk, Level of nitric oxide-dependent cGMP in patients with migraine. Cephalalgia, 1998. 18(9): p. 631-4.

74. Smith, J.M., et al., Physiological studies of cortical spreading depression. Biol Rev Camb Philos Soc, 2006. 81(4): p. 457-81.

75. Takano, T., et al., Cortical spreading depression causes and coincides with tissue hypoxia. Nat Neurosci, 2007. 10(6): p. 754-62.

76. Bolay, H., D. Vuralli, and P.J. Goadsby, Aura and Head pain: relationship and gaps in the translational models. J Headache Pain, 2019. 20(1): p. 94.

77. Bolay, H. and M.A. Moskowitz, The emerging importance of cortical spreading depression in migraine headache. Rev Neurol (Paris), 2005. 161(6-7): p. 655-7.

78. Borkum, J.M., The Migraine Attack as a Homeostatic, Neuroprotective Response to Brain Oxidative Stress: Preliminary Evidence for a Theory. Headache, 2018. 58(1): p. 118-135.

79. Mauskop, A. and J. Varughese, Why all migraine patients should be treated with magnesium. J Neural Transm (Vienna), 2012. 119(5): p. 575-9.

80. Pietrobon, D. and J. Striessnig, Neurobiology of migraine. Nat Rev Neurosci, 2003. 4(5): p. 386-98. 
81. Stankewitz, A. and A. May, The phenomenon of changes in cortical excitability in migraine is not migraine-specific--a unifying thesis. Pain, 2009. 145(1-2): p. 14-7.

82. Stankewitz, A. and A. May, Cortical excitability and migraine. Cephalalgia, 2007. 27(12): p. 1454-6.

83. Cosentino, G., et al., Cyclical changes of cortical excitability and metaplasticity in migraine: evidence from a repetitive transcranial magnetic stimulation study. Pain, 2014. 155(6): p. 1070-8.

84. Bednarczyk, E.M., et al., Global cerebral blood flow, blood volume, and oxygen metabolism in patients with migraine headache. Neurology, 1998. 50(6): p. 1736-40.

85. Hodkinson, D.J., et al., Primary Somatosensory Cortices Contain Altered Patterns of Regional Cerebral Blood Flow in the Interictal Phase of Migraine. PLoS One, 2015. 10(9): p. e0137971.

86. Li, Z., et al., Altered periaqueductal gray resting state functional connectivity in migraine and the modulation effect of treatment. Sci Rep, 2016. 6: p. 20298.

87. Marciszewski, K.K., et al., Changes in Brainstem Pain Modulation Circuitry Function over the Migraine Cycle. J Neurosci, 2018. 38(49): p. 10479-10488.

88. Rocca, M.A., et al., Brain gray matter changes in migraine patients with T2-visible lesions: a 3-T MRI study. Stroke, 2006. 37(7): p. 1765-70.

89. May, A., New insights into headache: an update on functional and structural imaging findings. Nat Rev Neurol, 2009. 5(4): p. 199-209.

90. Valfre, W., et al., Voxel-based morphometry reveals gray matter abnormalities in migraine. Headache, 2008. 48(1): p. 109-17.

91. Schmitz, N., et al., Attack frequency and disease duration as indicators for brain damage in migraine. Headache, 2008. 48(7): p. 1044-55.

92. Liu, J., et al., Migraine-related gray matter and white matter changes at a 1-year follow-up evaluation. J Pain, 2013. 14(12): p. 1703-8.

93. Schmidt-Wilcke, T., et al., Subtle grey matter changes between migraine patients and healthy controls. Cephalalgia, 2008. 28(1): p. 1-4.

94. DaSilva, A.F., et al., Thickening in the somatosensory cortex of patients with migraine. Neurology, 2007. 69(21): p. 1990-5. 
95. Granziera, C., et al., Anatomical alterations of the visual motion processing network in migraine with and without aura. PLoS Med, 2006. 3(10): p. e402.

96. Maleki, N., et al., Her versus his migraine: multiple sex differences in brain function and structure. Brain, 2012. 135(Pt 8): p. 2546-59.

97. Yuan, K., et al., Altered structure and resting-state functional connectivity of the basal ganglia in migraine patients without aura. J Pain, 2013. 14(8): p. 836-44.

98. Moulton, E.A., et al., Painful heat reveals hyperexcitability of the temporal pole in interictal and ictal migraine States. Cereb Cortex, 2011. 21(2): p. 435-48.

99. Chabriat, H. and M.G. Bousser, Neuropsychiatric manifestations in CADASIL. Dialogues Clin Neurosci, 2007. 9(2): p. 199-208.

100. Maleki, N., et al., Migraine attacks the Basal Ganglia. Mol Pain, 2011. 7: p. 71.

101. Maleki, N., et al., Common hippocampal structural and functional changes in migraine. Brain Struct Funct, 2013. 218(4): p. 903-12.

102. Schwedt, T.J., et al., Enhanced pain-induced activity of pain-processing regions in a case-control study of episodic migraine. Cephalalgia, 2014. 34(12): p. 947-58.

103. Schwedt, T.J. and D.W. Dodick, Advanced neuroimaging of migraine. Lancet Neurol, 2009. 8(6): p. 560-8.

104. Colombo, B., D. Dalla Libera, and G. Comi, Brain white matter lesions in migraine: what's the meaning? Neurol Sci, 2011. 32 Suppl 1: p. S37-40.

105. Szabo, N., et al., White matter microstructural alterations in migraine: a diffusionweighted MRI study. Pain, 2012. 153(3): p. 651-6.

106. Rocca, M.A., et al., Evidence for cortical functional changes in patients with migraine and white matter abnormalities on conventional and diffusion tensor magnetic resonance imaging. Stroke, 2003. 34(3): p. 665-70.

107. Negm, M., et al., Relation between migraine pattern and white matter hyperintensities in brain magnetic resonance imaging. Egypt J Neurol Psychiatr Neurosurg, 2018. 54(1): p. 24.

108. Leijdekkers, M.L., et al., Migraine patients cognitively impaired? Headache, 1990. 30(6): p. 352-8. 
109. Mulder, E.J., et al., Interictal and postictal cognitive changes in migraine. Cephalalgia, 1999. 19(6): p. 557-65; discussion 541.

110. Le Pira, F., et al., Memory disturbances in migraine with and without aura: a strategy problem? Cephalalgia, 2000. 20(5): p. 475-8.

111. Calandre, E.P., et al., Cognitive disturbances and regional cerebral blood flow abnormalities in migraine patients: their relationship with the clinical manifestations of the illness. Cephalalgia, 2002. 22(4): p. 291-302.

112. Gil-Gouveia, R. and I.P. Martins, Cognition and Cognitive Impairment in Migraine. Curr Pain Headache Rep, 2019. 23(11): p. 84.

113. Pearson, A.J., et al., Cognitive function is not impaired in people with a long history of migraine: a blinded study. Cephalalgia, 2006. 26(1): p. 74-80.

114. Shepherd, A.J., H.M. Beaumont, and T.J. Hine, Motion processing deficits in migraine are related to contrast sensitivity. Cephalalgia, 2012. 32(7): p. 554-70.

115. Antal, A., et al., Altered motion perception in migraineurs: evidence for interictal cortical hyperexcitability. Cephalalgia, 2005. 25(10): p. 788-94.

116. McColl, S.L. and F. Wilkinson, Visual contrast gain control in migraine: measures of visual cortical excitability and inhibition. Cephalalgia, 2000. 20(2): p. 74-84.

117. Braunitzer, G., et al., Is the development of visual contrast sensitivity impaired in children with migraine? An exploratory study. Cephalalgia, 2010. 30(8): p. 991-5.

118. Braunitzer, G., et al., Development of visual contour integration in children with migraine without aura. Cephalalgia, 2011. 31(9): p. 1048-56.

119. Braunitzer, G., et al., Delayed development of visual motion processing in childhood migraine. Cephalalgia, 2012. 32(6): p. 492-6.

120. D'Andrea, G., et al., Personality and memory in childhood migraine. Cephalalgia, 1989. 9(1): p. 25-8.

121. Parisi, P., et al., Headache and cognitive profile in children: a cross-sectional controlled study. J Headache Pain, 2010. 11(1): p. 45-51.

122. Waldie, K.E., et al., Migraine and cognitive function: a life-course study. Neurology, 2002. 59(6): p. 904-8. 
123. Petrusic, I., et al., Addenbrooke's cognitive examination test for brief cognitive assessment of adolescents suffering from migraine with aura. Acta Neurol Belg, 2017. 117(1): p. 97-102.

124. Oze, A., et al., Acquired equivalence and related memory processes in migraine without aura. Cephalalgia, 2017. 37(6): p. 532-540.

125. Vuralli, D., C. Ayata, and H. Bolay, Cognitive dysfunction and migraine. J Headache Pain, 2018. 19(1): p. 109.

126. Morton, R.E., P.D. St John, and S.L. Tyas, Migraine and the risk of all-cause dementia, Alzheimer's disease, and vascular dementia: A prospective cohort study in community-dwelling older adults. Int J Geriatr Psychiatry, 2019. 34(11): p. 16671676.

127. Eordegh, G., et al., Multisensory guided associative learning in healthy humans. PLoS One, 2019. 14(3): p. e0213094.

128. Puszta, A., et al., Power-spectra and cross-frequency coupling changes in visual and Audio-visual acquired equivalence learning. Sci Rep, 2019. 9(1): p. 9444.

129. Puszta, A., et al., Cortical Power-Density Changes of Different Frequency Bands in Visually Guided Associative Learning: A Human EEG-Study. Front Hum Neurosci, 2018. 12: p. 188.

130. Braunitzer, G., et al., The development of acquired equivalence from childhood to adulthood-A cross-sectional study of 265 subjects. PLoS One, 2017. 12(6): p. $\mathrm{e} 0179525$.

131. Burstein, R., R. Noseda, and D. Borsook, Migraine: multiple processes, complex pathophysiology. J Neurosci, 2015. 35(17): p. 6619-29.

132. Chong, C.D., et al., Structural alterations of the brainstem in migraine. Neuroimage Clin, 2017. 13: p. 223-227.

133. Petrusic, I., M. Dakovic, and J. Zidverc-Trajkovic, Subcortical Volume Changes in Migraine with Aura. J Clin Neurol, 2019. 15(4): p. 448-453.

134. Liu, H.Y., et al., Hippocampus and amygdala volume in relation to migraine frequency and prognosis. Cephalalgia, 2017. 37(14): p. 1329-1336. 
135. Larsen, B. and B. Luna, In vivo evidence of neurophysiological maturation of the human adolescent striatum. Dev Cogn Neurosci, 2015. 12: p. 74-85.

136. Frassinetti, F., N. Bolognini, and E. Ladavas, Enhancement of visual perception by crossmodal visuo-auditory interaction. Exp Brain Res, 2002. 147(3): p. 332-43.

137. Fort, A., et al., Dynamics of cortico-subcortical cross-modal operations involved in audio-visual object detection in humans. Cereb Cortex, 2002. 12(10): p. 1031-9.

138. Suied, C., N. Bonneel, and I. Viaud-Delmon, Integration of auditory and visual information in the recognition of realistic objects. Exp Brain Res, 2009. 194(1): p. 91102.

139. Chen, X., et al., The integration of facial and vocal cues during emotional change perception: EEG markers. Soc Cogn Affect Neurosci, 2016. 11(7): p. 1152-61.

140. Love, S.A., F.E. Pollick, and M. Latinus, Cerebral correlates and statistical criteria of cross-modal face and voice integration. Seeing Perceiving, 2011. 24(4): p. 351-67.

141. Joassin, F., et al., Cross-modal interactions between human faces and voices involved in person recognition. Cortex, 2011. 47(3): p. 367-76.

142. Tukey J. Exploratory Data Analysis. Boston, Massachusetts: Addison-Wesley Publishing Company, 1977. 
Zsófia Giricz, Ákos Pertich, Attila Őze, András Puszta, Ágnes Fehér, Gabriella Eördegh, Jenő Kóbor, Katalin Bihari, Éva Pálinkás, Gábor Braunitzer, Attila Nagy (2020) Visually guided associative learning in pediatric and adult migraine without aura. DOI: 10.1177/0333102420958388 